\title{
Exploring the generalized loosely bound Skyrme model
}

\author{
Sven Bjarke Gudnason \\ Department of Physics, and Research and Education Center for Natural Sciences, Keio University, \\ Hiyoshi 4-1-1, Yokohama, Kanagawa 223-8521, Japan
}

(Received 7 June 2018; published 28 November 2018)

\begin{abstract}
The Skyrme model is extended with a sextic derivative term, called the BPS-Skyrme term, and a repulsive potential term, called the loosely bound potential. A large part of the model's parameter space is studied for the 4-Skyrmion, which corresponds to the helium-4 nucleus, and emphasis is put on preserving as much of the platonic symmetries as possible while reducing the binding energies. We reach classical binding energies for helium- 4 as low as $0.2 \%$, while retaining the cubic symmetry of the 4-Skyrmion, and after taking into account the quantum mass correction to the nucleon due to spin/isospin quantization, we get total binding energies as low as $3.6 \%$ - still with the cubic symmetry intact.
\end{abstract}

DOI: 10.1103/PhysRevD.98.096018

\section{INTRODUCTION}

The Skyrme model is an interesting approach to nuclear physics that is related to fundamental physics; i.e., it is a field theory [1,2]. Indeed, the soliton in the model is identified with the baryon in the large- $N$ limit of QCD $[3,4]$. The soliton is called the Skyrmion. The most interesting aspect of the Skyrmions is that, although a single Skyrmion is identified with a single nucleon, multiSkyrmion solutions that can be identified with nuclei of higher baryon numbers exist. This fact distinguishes the Skyrme model from basically all other approaches to nuclear physics; the nuclei are no longer bound states of interacting point particles. What is more interesting is that, since even the single Skyrmion is a spatially extended object (as opposed to a point particle), the multi-Skyrmions become extended objects with certain platonic symmetries [5]. A particular useful ansatz for light nuclei was found using a rational map [6]. For larger nuclei, however, there is some consensus that when a pion mass term is included in the model they are made of $B / 4$ cubes-akin somewhat to the alpha particle model of nuclei [7-11] —up to small deformations.

A long-standing problem of the Skyrme model as a model for nuclei is that the multi-Skyrmions are too strongly bound; their binding energies are about 1 order of magnitude larger than what is measured in nuclei experimentally. One approach to solving this problem is

\footnotetext{
gudnason@keio.jp
}

Published by the American Physical Society under the terms of the Creative Commons Attribution 4.0 International license. Further distribution of this work must maintain attribution to the author(s) and the published article's title, journal citation, and DOI. Funded by SCOAP. based on modifying the Skyrme model such that the classical solutions can come close to a BPS-like energy bound, in which case the classical energy is approximately proportional to the topological charge. Hence, if such a bound could be saturated, then classically the binding energy would vanish exactly. In the last decade, this approach has been taken in three different directions: the vector meson Skyrme model [12-14], the BPS-Skyrme model $[15,16]$, and the lightly/loosely bound Skyrme models [17-19].

The vector meson Skyrme model is inspired by approximate Skyrmion solutions obtained from instanton holonomies [20], and it is found that the instanton holonomy becomes an exact solution in the limit where an infinite tower of vector mesons is included; the complete theory can be described simply as a 4+1-dimensional Yang-Mills (YM) theory in flat spacetime [12]. The instanton is a halfBPS state in the YM theory, and the Skyrmion saturates the BPS bound if the theory is not truncated. The standard Skyrme model is at the other end of the scale; all vector mesons have been stripped off, leaving behind just the pions. The model is in very close relation to holography, although the discrete spectrum of vector mesons is due to truncation of the theory and not due to an intrinsic curvature of the background spacetime [12]. Indeed, in the SakaiSugimoto model, the standard Skyrme model comes out as the low-energy action of the zero modes [21]. The sextic term, which we shall discuss shortly in a different context, also comes out naturally by integrating out the first vector meson in the Sakai-Sugimoto model [22].

The BPS-Skyrme model is based on a drastic modification of the Skyrme model: remove the original terms, and replace them with a sextic term, which we shall call the BPS-Skyrme term, and a potential $[15,16]$. The model has the advantage of simplicity in the following sense: the 
model in this limit is not only a BPS theory-in the sense of being able to saturate a BPS-like energy bound-but it is also integrable. That is, a large class of exact analytic solutions has been obtained. The disadvantage is that the kinetic term and the Skyrme term have to be quite suppressed in order for the classical binding energy to be of the order of magnitude seen in experimental data. That issue is twofold; the first problem is that we would like to maintain the coefficient of the kinetic term (the pion decay constant) and pion mass of the order of the measured values in the pion vacuum. The second problem is of a more technical nature; close to the BPS limit, the coefficient of the kinetic term $\left(c_{2}\right)$ is very small, and hence there are certain points/lines in the Skyrmion solutions where the solution can afford to have very large field derivatives - of the order of $1 / \sqrt{c_{2}}$. In Ref. [18], the order of magnitude of $c_{2}$ for the classical binding energies to be in the ballpark of the experimental values was estimated to be around $c_{2} \sim 0.01$, whereas their numerics was trustable only down to about $c_{2} \sim 0.2$.

The lightly bound Skyrme model is based on an energy bound $[17,23]$ for the Skyrme term and a potential to the fourth power. Although this model has a saturable solution in the 1-Skyrmion sector, no solutions saturate the bound for higher topological degrees. Nevertheless, it turns out that, although the solutions do not saturate the bound for higher topological degrees, they can come quite close to the bound, which in turn yields a small classical binding energy [18]. The lightly bound model can indeed reduce the binding energies, but it comes with a price; long before realistic binding energies are reached, the platonic symmetries of the compact Skyrmions are lost, and the potential has the effect of pushing out identifiable 1-Skyrmions, which remain only very weakly bound. This limit was the inspiration for a simplified kind of Skyrme model, called the point particle model of lightly bound Skyrmions [24]. Said limit can also be obtained naturally in the SakaiSugimoto model by considering the strong 't Hooft coupling limit [25].

In Ref. [19], we compared the potential made of the standard pion mass term to the fourth power and the same potential to the second power; we call them the lightly bound and the loosely bound potentials, respectively. It turns out that the loosely bound potential has the same repulsive effect as the lightly bound potential does, but the Skyrmions retain their platonic symmetries down to smaller binding energies for the loosely bound potential as compared to the lightly bound one. In Ref. [26], we further established that if the potential is treated as a polynomial in $\sigma=\frac{1}{2} \operatorname{Tr}[U]$, where $U$ is the chiral field, then to second order the loosely bound potential is the potential that can reduce the binding energy the most while preserving platonic symmetries of the Skyrmions.

In Ref. [27], we expanded the model by making a hybrid model out of the BPS-Skyrme-type models and the loosely bound model. Let us define the generalized Skyrme model as the standard Skyrme model with the addition of the sextic BPS-Skyrme term. Thus, in Ref. [27], we studied the generalized Skyrme model with the pion mass term and the loosely bound potential. More precisely, we studied the model using the rational map ansatz for the 4-Skyrmion in the regime where the coefficients for the BPS-Skyrme term, $c_{6}$, and the loosely bound potential, $m_{2}$, were both taken to be small (i.e., smaller than or equal to 1). Physical effects on the observables of the model could readily be extracted without the effort of full numerical partial differential equation (PDE) calculations. Of course, that compromise had the consequence that we could not detect the change of symmetry in the Skyrmion solutions, and hence we investigated only a very restricted part of the parameter space.

In this paper, we perform the full PDE calculations for the 4-Skyrmion in the generalized Skyrme model with the loosely bound potential and a standard pion mass term, for small values of the coefficient of the BPS-Skyrme term and up to large values of the mass parameter of the loosely bound potential; see Fig. 1. In particular, in this paper, we study the following region of parameter space: $c_{6} \in[0,1]$ and $m_{2} \in[0,6]$. Note that it is $m_{2}^{2}$ that enters the Lagrangian, and hence $6^{2}=36$ is much larger than the other coefficients in the Lagrangian (which are all of order 1). In fact, we are close to the limit of how far we can push $m_{2}$ with the current numerical codes. We have not considered the direction of large $c_{6}$ in this work, as it will not reduce the binding energy unless we also turn on a large coefficient of the potential. That situation, however, yields two possibilities: either we do not go beyond the limit where the numerics becomes difficult as discussed above or one has to take the near-BPS limit carefully, which will require overcoming further technical obstacles than dealt with here. Nevertheless, in the part of parameter space we have studied in this paper, we are able to obtain a classical binding energy of the 4-Skyrmion as low as $0.2 \%$ about a factor of 4 smaller than the experimental value

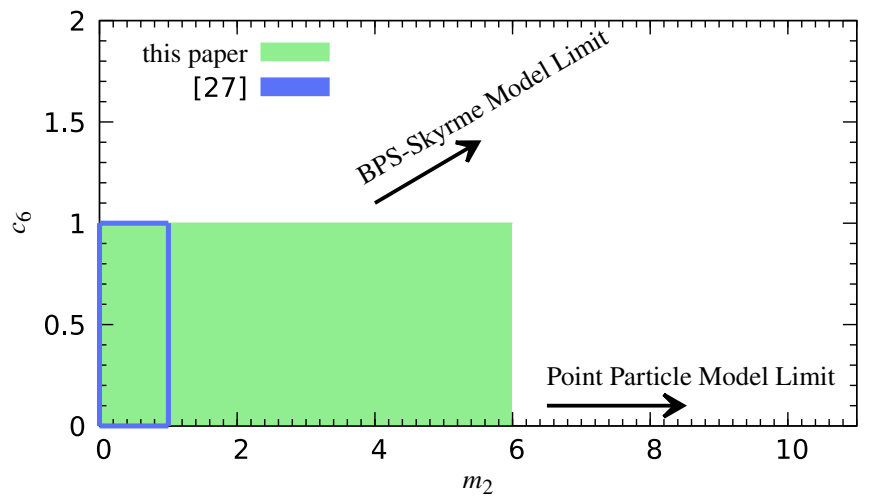

FIG. 1. Parameter space of the model explored in this paper (green) as well as what was studied in Ref. [27] (region inside the blue box). The BSML is defined as $c_{6} \propto m_{2} \rightarrow \infty$, and the PPML is $c_{6}=0, m_{2} \rightarrow \infty$. 
for helium-4. After taking into account the quantum correction to the mass of the nucleon due to the spin contribution (as it is a spin- $-\frac{1}{2}$ state in the ground state), the binding energy increases to about 3.6\%-about a factor of 4.5 too large. This fact suggests that we cannot leave out further quantum corrections to the nuclear masses; we have to take into account quantum corrections due to massive modes [28], also called vibrational modes; see, e.g., Refs. [10,29,30].

The question remains how large a total contribution the inclusion of the quantum corrections due to the massive modes will give. It has been assumed all along that the quantization of solitons can consistently be made in a semiclassical fashion, in which the quantum corrections are small compared to the (large) mass scale of the soliton. This expectation is based on the assumption that the fluctuation spectrum is weakly coupled, even though the soliton is inherently a nonperturbative object. The simplest example is to consider the mass correction to the kink in the $\lambda \phi^{4}$ model in $1+1$ dimensions,

$$
\mathcal{L}^{\text {kink }}=-\frac{1}{2}\left(\partial_{\mu} \phi\right)^{2}-\frac{\lambda}{4 \hbar^{3}}\left(\phi^{2}-\frac{\hbar m^{2}}{\lambda}\right)^{2} .
$$

We can estimate the kink mass with the following back-ofan-envelope estimate: we rescale the length scale $x^{\mu} \rightarrow$ $\hbar x^{\mu} / m$ and rescale the field $\phi \rightarrow \sqrt{\frac{\hbar}{\lambda}} m \phi$; the Lagrangian density is now dimensionless with an overall dimensionful prefactor of $\frac{m^{4}}{\hbar \lambda}$. Assuming the kink exists, its mass must be an order 1 number times $\frac{m^{3}}{\lambda}$, where the $\hbar / m$ came from integrating over $x^{1}$. Considering now the quantum correction due to massive modes, one obtains an order-1 number times $\hbar \omega$, where $\omega^{2}$ is the curvature of the effective potential created by the kink solution [31]. That is, the eigenvalue of the fluctuation around the kink is $\omega^{2} \sim m^{2} / \hbar^{2}$, and it follows in the harmonic approximation that the quantum energy is $\hbar \omega \sim m$. To realize this, it suffices to note that the second variation of the potential with respect to the field is $-\frac{m^{2}}{\hbar^{2}}+3 \frac{\lambda}{\hbar^{3}} \phi_{\text {soliton }}^{2}$ and that the soliton solution is proportional to $\hbar^{\frac{1}{2}} \lambda^{-\frac{1}{2}} m$; the resultant effective potential for the fluctuations is thus independent of $\lambda$. In this example, the mass dimensions of $m$ and $\lambda$ are 1 and 2 , respectively. If $\lambda \ll m^{2}$, then the perturbation series makes sense, and furthermore the quantum correction is much smaller than the classical contribution

$$
M_{\text {classical }}+\delta M \propto \frac{m^{3}}{\lambda}\left(1+\mathcal{O}\left(\frac{\lambda}{m^{2}}\right)\right) .
$$

The situation is more complicated in the case of the threedimensional Skyrmions than in the case of simple onedimensional kinks (which are integrable). First of all, to the best of our knowledge, it is not known how weakly coupled the fluctuation spectrum really is. Comparing to the kinks, the question would be how small $\lambda^{\text {eff }}$ is for the Skyrmions.

The scope in this paper, however, will be to focus on reducing the classical binding energy of the Skyrmions under the constraint of preserving as much symmetry of the original Skyrmions as possible. This is thus in the spirit of assuming that the fluctuation spectrum of the Skyrmions is weakly coupled and thus the classical mass is the largest contribution to the mass by far; the zero-mode quantization gives the most important quantum corrections, and the remaining modes give corrections to the mass of the order of magnitude of the zero-mode contributions or less. The reason for preserving as much symmetry as possible is first of all to be able to keep some of the phenomenological successes of the Skyrme model already obtained, such as the Hoyle state and its corresponding rotational band having a slope a factor of 2.5 lower than that of the ground state [9]. Taking into account the vibrational spectrum of the 4-Skyrmions vibrating between a flat square configuration and a tetrahedral arrangement was crucial in obtaining the right spectrum of oxygen-16, having a large energy splitting between states of the same spins and opposite parity [10]. Both of these results would fall apart if the 4-Skyrmion were to lose its cubic symmetry. Finally, and perhaps even more importantly, the larger the symmetry, the better the chances are that the symmetry can eliminate unwanted degeneracies, e.g., the parity doubling found in the $B=5$ cluster system in the model of Ref. [30].

The paper is organized as follows. In the next section, we will introduce the model, define the observables, and finally propose an order parameter for a quantitative measure of the symmetry change. In Sec. III, we will present the numerical results. Finally, Sec. IV concludes the paper with a discussion of the results and what to do next.

\section{MODEL}

The model we study in this paper is the generalized Skyrme model — consisting of a kinetic term, the Skyrme term, and the BPS-Skyrme term - with a pion mass term and the so-called loosely bound potential. In physical units, we have

$$
\mathcal{L}=\frac{\tilde{f}_{\pi}^{2}}{4} \mathcal{L}_{2}+\frac{1}{e^{2}} \mathcal{L}_{4}+\frac{4 c_{2} c_{6}}{c_{4}^{2} e^{4} \tilde{f}_{\pi}^{2}} \mathcal{L}_{6}-\frac{\tilde{m}_{\pi}^{2} \tilde{f}_{\pi}^{2}}{4 m_{1}^{2}} V,
$$

where the kinetic term, the Skyrme term [1,2], and the BPS-Skyrme term $[15,16]$ are given by

$$
\begin{aligned}
& \mathcal{L}_{2}=\frac{1}{4} \operatorname{Tr}\left(L_{\mu} L^{\mu}\right), \\
& \mathcal{L}_{4}=\frac{1}{32} \operatorname{Tr}\left(\left[L_{\mu}, L_{\nu}\right]\left[L^{\mu}, L^{\nu}\right]\right), \\
& \mathcal{L}_{6}=\frac{1}{144} \eta_{\mu \mu^{\prime}}\left(\epsilon^{\mu \nu \rho \sigma} \operatorname{Tr}\left[L_{\nu} L_{\rho} L_{\sigma}\right]\right)\left(\epsilon^{\mu^{\prime} \nu^{\prime} \rho^{\prime} \sigma^{\prime}} \operatorname{Tr}\left[L_{\nu^{\prime}} L_{\rho^{\prime}} L_{\sigma^{\prime}}\right]\right),
\end{aligned}
$$


with the left-invariant current defined as

$$
L_{\mu} \equiv U^{\dagger} \partial_{\mu} U
$$

in terms of the chiral Lagrangian or Skyrme field $U$, which is related to the pions as

$$
U=\mathbf{1}_{2} \sigma+i \tau \cdot \boldsymbol{\pi},
$$

with the nonlinear sigma model constraint $\operatorname{det} U=1$ or $\sigma^{2}+\boldsymbol{\pi} \cdot \boldsymbol{\pi}=1$ and $\boldsymbol{\tau}$ being a 3 -vector of the Pauli matrices. The greek indices $\mu, \nu, \rho, \sigma=0,1,2,3$ denote spacetime indices, and we will use the mostly positive metric signature throughout the paper.

We will now switch to (dimensionless) Skyrme units following Refs. [26,27] and denote the quantities having physical units with a tilde. In particular, for the energy and length scale, we will take $\tilde{E}=\tilde{\lambda} E$ and $\tilde{x}^{i}=\tilde{\mu} x^{i}$, respectively, where we have the units [27]

$$
\tilde{\lambda}=\frac{\tilde{f}_{\pi}}{2 e \sqrt{c_{2} c_{4}}}, \quad \tilde{\mu}=\sqrt{\frac{c_{2}}{c_{4}}} \frac{2}{e \tilde{f}_{\pi}},
$$

and finally the pion mass in physical units [27]

$$
\tilde{m}_{\pi}=\frac{\sqrt{c_{4}}}{2 c_{2}} e \tilde{f}_{\pi} m_{1} .
$$

Hence, in dimensionless units, the Lagrangian (3) reads

$$
\mathcal{L}=c_{2} \mathcal{L}_{2}+c_{4} \mathcal{L}_{4}+c_{6} \mathcal{L}-V .
$$

For a positive definite energy density, we require $c_{2}>0$, $c_{4}>0$ and $c_{6} \geq 0 .[32]$.

The potential we will consider in this paper is due to the results of Ref. [19], which showed that the pion mass term squared lowers the binding energy further than the pion mass term to the fourth power while keeping the symmetries of the 4-Skyrmion. We will also include the standard pion mass term, and thus the total potential is

$$
V=V_{1}+V_{2},
$$

where we have defined

$$
V_{n} \equiv \frac{1}{n} m_{n}^{2}(1-\sigma)^{n},
$$

and $\sigma=\frac{1}{2} \operatorname{Tr}[U]$. Only $V_{1}$ gives a contribution to the pion mass. Both $V_{1}$ and $V_{2}$ break explicitly the chiral symmetry $\mathrm{SU}(2)_{\mathrm{L}} \times \mathrm{SU}(2)_{\mathrm{R}}$ to the diagonal $\mathrm{SU}(2)_{\mathrm{L}+\mathrm{R}}$. The target space is thus $\mathrm{SU}(2)_{\mathrm{L}} \times \mathrm{SU}(2)_{\mathrm{R}} / \mathrm{SU}(2)_{\mathrm{L}+\mathrm{R}} \simeq \mathrm{SU}(2) \simeq S^{3}$. Since the Skyrmion, which is identified with the baryon, is a texture [36], it is characterized by the topological degree, $B$,

$$
\pi_{3}\left(S^{3}\right)=\mathbb{Z} \ni B
$$

where $B$ is called the baryon number. The baryon number or topological degree of a Skyrmion configuration can be calculated by

$B=\frac{1}{2 \pi^{2}} \int d^{3} x \mathcal{B}^{0}, \quad \mathcal{B}^{\mu}=-\frac{1}{12} \epsilon^{\mu \nu \rho \sigma} \operatorname{Tr}\left(L_{\nu} L_{\rho} L_{\sigma}\right)$.

Throughout the paper, we will denote Skyrmions of degree $B$ as $B$-Skyrmions.

Finally, for the numerical calculations, we have to settle on a choice of normalization of the units, and we follow that of Refs. [19,26,27],

$$
c_{2}=\frac{1}{4}, \quad c_{4}=1,
$$

and hence the energies and lengths are given in units of $\tilde{f}_{\pi} / e$ and $1 /\left(e \tilde{f}_{\pi}\right)$, respectively, while the physical pion mass is given by

$$
\tilde{m}_{\pi}=\left.\frac{\sqrt{c_{4}}}{2 c_{2}} e \tilde{f}_{\pi} \sqrt{-\frac{\partial V}{\partial \sigma}}\right|_{\sigma=1}=2 e \tilde{f}_{\pi} m_{1},
$$

where we have used the coefficients (16). In this paper, we will use $m_{1}=1 / 4$ [19].

\section{A. Observables}

In this section, we will list the observables to be measured in the numerical calculations. Since they are greatly overlapping with our previous studies, we will only review them briefly here and refer to Ref. [27] for details. As in Refs. [26,27], we will only consider the 4-Skyrmion in this paper, as it plays a unique role in the alpha-particle interpretation of the Skyrme model and it is the building block of the lattice structure appearing for large nuclei [7-11]. More importantly, it is where to look for the change in symmetry that inevitably kicks in for strongly repulsive potentials; see, e.g., the point particle model $[18,24]$ and also Ref. [19].

As usual, we are interested in the classical and spin/ isospin quantum-corrected binding energies. The energy of the 1-Skyrmion is obtained by minimizing the static energy corresponding to (minus) the Lagrangian (11) for the hedgehog

$$
U=\mathbf{1}_{2} \cos f(r)+i \hat{\mathbf{x}} \cdot \boldsymbol{\tau} \sin f(r),
$$

where $\hat{\mathbf{x}} \equiv \mathbf{x} / r$ is the unit 3 -vector at the origin and $r=\sqrt{\mathbf{x} \cdot \mathbf{x}}$ is the radial coordinate. We will call the energy of the $B$-Skyrmion $E_{B}$. As the initial condition for the 4-Skyrmion, we will use the rational map ansatz [6]

$$
U=\mathbf{1}_{2} \cos f(r)+i \mathbf{n}_{R} \cdot \boldsymbol{\tau} \sin f(r),
$$




$$
\begin{aligned}
\mathbf{n}_{R} & =\left(\frac{R+\bar{R}}{1+|R|^{2}}, \frac{i(\bar{R}-R)}{1+|R|^{2}}, \frac{1-|R|^{2}}{1+|R|^{2}}\right), \\
R(z) & =\frac{z^{4}+2 \sqrt{3} i z^{2}+1}{z^{4}-2 \sqrt{3} i z^{2}+1},
\end{aligned}
$$

for the $c_{6}=0, m_{2}=0$ solution, and $z=e^{i \phi} \tan \left(\frac{\theta}{2}\right)$ is the Riemann sphere coordinate. Once a numerical solution has been obtained, we can calculate the classical relative binding energy (CRBE) of the 4-Skyrmion as

$$
\delta_{4}=1-\frac{E_{4}}{4 E_{1}}
$$

and the quantum-corrected relative binding energy (QRBE) as

$$
\delta_{4}^{\mathrm{tot}}=1-\frac{E_{4}}{4\left(E_{1}+\epsilon_{1}\right)},
$$

where $\epsilon_{1}$ is the quantum correction due to the isospin quantization of the 1-Skyrmion. The CRBE (22) is independent of the physical units and thus independent of the calibration of the model. The QRBE (23), on the other hand, after factoring out the energy units, still depends on the Skyrme coupling $e$.

Calibrating the Skyrme-like models can be done in many ways, and often ways are invented to minimize the problem of overbinding by compensating with a better calibration. In this paper, we will not turn to the calibration for compensating the overbinding but try to reduce the binding energy by varying the parameters of the Lagrangian (11). Thus, we will stick with a simple calibration in which we set the mass and size of the 4-Skyrmion to those of helium-4. To calculate the electric charge radius of the 4-Skyrmion, we note that the ground state of helium- 4 is an isospin- 0 state, and thus the charge radius in the Skyrme model is given entirely by the baryon charge radius [27]

$$
r_{4}^{2}=r_{4, E}^{2}=r_{4, B}^{2}=\frac{1}{8 \pi^{2}} \int d^{3} x r^{2} \mathcal{B}^{0} .
$$

The calibration now reads [27]

$$
\begin{gathered}
\tilde{f}_{\pi}=2 \sqrt{c_{2}} \sqrt{\frac{r_{4} \tilde{M}_{{ }^{4} \mathrm{He}}}{\tilde{r}_{4} \mathrm{He} E_{4}}}=\sqrt{\frac{r_{4} \tilde{M}_{{ }^{4} \mathrm{He}}}{\tilde{r}_{4} E_{\mathrm{He}} E_{4}}}, \\
e=\frac{1}{\sqrt{c_{4}}} \sqrt{\frac{r_{4} E_{4}}{\tilde{r}_{{ } \mathrm{He}} \tilde{M}_{{ }^{4} \mathrm{He}}}}=\sqrt{\frac{r_{4} E_{4}}{\tilde{r}_{{ }^{4} \mathrm{He}} \tilde{M}_{{ }^{4} \mathrm{He}}}}
\end{gathered}
$$

where in the latter expressions, we plugged in the normalization (16), and the experimental data used here are $\tilde{M}_{{ }^{H} \mathrm{He}}=3727 \mathrm{MeV}$ and $\tilde{r}_{{ }^{4} \mathrm{He}}=8.492 \times 10^{-3} \mathrm{MeV}^{-1}$.

With the calibration in place, we can now determine the quantum correction to the mass of the 1-Skyrmion due to (spin/)isospin quantization [with the normalization (16)]

$$
\begin{aligned}
\tilde{m}_{N} & \equiv \tilde{M}_{1}=\frac{\tilde{f}_{\pi}}{e}\left(E_{1}+\epsilon_{1}\right)=\tilde{E}_{1}+\tilde{\epsilon}_{1}, \\
\epsilon_{1} & =\frac{e^{4}}{2 \Lambda} J(J+1),
\end{aligned}
$$

where $\Lambda$ is the diagonal component of the isospin inertia tensor for the 1-Skyrmion, $U_{i j}=\Lambda \delta^{i j}$, where $U_{i j}$ is given in the next section in Eq. (33). For the hedgehog ansatz (18), the expression for $\Lambda$ reads

$\Lambda=\frac{8 \pi}{3} \int d r r^{2} \sin ^{2} f\left(c_{2}+c_{4} f_{r}^{2}+\frac{c_{4}}{r^{2}} \sin ^{2} f+\frac{2 c_{6} \sin ^{2}(f) f_{r}^{2}}{r^{2}}\right)$.

Finally, for the ground state of the proton, $J=\frac{1}{2}$, and thus $\epsilon_{1}=\frac{3 e^{4}}{8 \Lambda}$. We will also consider the $\Delta$ resonance as a spin- $\frac{3}{2}$ excitation of the 1-Skyrmion [37], yielding

$$
\tilde{m}_{\Delta}=\frac{\tilde{f}_{\pi}}{e}\left(E_{1}+5 \epsilon_{1}\right) .
$$

The $\Delta$ resonance is nevertheless problematic in the Skyrme model; see the discussion.

As the ground state of helium- 4 is a spin- 0 , isospin- 0 state, there is no quantum correction to the mass due to zero-mode quantization (although there are corrections due to massive modes; see the discussion).

Finally, we will consider the electric charge radius of the proton and the axial coupling. The details and tensor expressions are given in Ref. [27], and we will just state the final results here,

$$
\begin{aligned}
r_{1, E}^{2} & =\frac{1}{2} r_{1, B}^{2}+\frac{\int d r r^{2}\left(c_{2} r^{2} \sin ^{2} f+c_{4} \sin ^{2}(f)\left(\sin ^{2} f+r^{2} f_{r}^{2}\right)+2 c_{6} \sin ^{4}(f) f_{r}^{2}\right)}{2 \int d r\left(c_{2} r^{2} \sin ^{2} f+c_{4} \sin ^{2}(f)\left(\sin ^{2} f+r^{2} f_{r}^{2}\right)+2 c_{6} \sin ^{4}(f) f_{r}^{2}\right)}, \\
g_{A} & =-\frac{4 \pi}{3} \int d r r\left[c_{2}\left(\sin 2 f+r f_{r}\right)+c_{4}\left(\frac{\sin ^{2} f \sin 2 f}{r^{2}}+\frac{2 \sin ^{2}(f) f_{r}}{r}+\sin (2 f) f_{r}^{2}\right)+\frac{2 c_{6} \sin ^{2} f}{r^{2}}\left(\frac{\sin ^{2}(f) f_{r}}{r}+\sin (2 f) f_{r}^{2}\right)\right],
\end{aligned}
$$


where the baryon charge radius is

$$
r_{1, B}^{2}=-\frac{2}{\pi} \int d r r^{2} \sin ^{2}(f) f_{r}
$$

and the axial coupling in physical units is given by

$$
\tilde{g}_{A}=\frac{g_{A}}{c_{4} e^{2}}=\frac{g_{A}}{e^{2}}
$$

which is obtained by multiplying the dimensionless expression by $\tilde{\lambda} \tilde{\mu}$ and in the last expression we have used the normalization (16).

Some geometric observables that we will calculate are the tensors of inertia corresponding to the spin and the isospin of the 4-Skyrmion. For the generalized model (3), they can be written as [38]

$$
\begin{aligned}
U_{i j}= & -\frac{1}{2} \int d^{3} x\left(c_{2} \operatorname{Tr}\left(T_{i} T_{j}\right)+\frac{c_{4}}{4} \operatorname{Tr}\left(\left[L_{k}, T_{i}\right]\left[L_{k}, T_{j}\right]\right)\right. \\
& \left.-\frac{c_{6}}{8} \operatorname{Tr}\left(T_{i}\left[L_{k}, L_{l}\right]\right) \operatorname{Tr}\left(T_{j}\left[L_{k}, L_{l}\right]\right)\right) \\
V_{i j}= & -\frac{1}{2} \int d^{3} x \epsilon^{i m n} \epsilon^{j p q} x^{m} x^{p}\left(c_{2} \operatorname{Tr}\left(L_{n} L_{q}\right)\right. \\
& +\frac{c_{4}}{4} \operatorname{Tr}\left(\left[L_{k}, L_{n}\right]\left[L_{k}, L_{q}\right]\right) \\
& \left.-\frac{c_{6}}{8} \operatorname{Tr}\left(L_{n}\left[L_{k}, L_{l}\right]\right) \operatorname{Tr}\left(L_{q}\left[L_{k}, L_{l}\right]\right)\right) \\
W_{i j}= & \frac{1}{2} \int d^{3} x \epsilon^{j m n} x^{m}\left(c_{2} \operatorname{Tr}\left(T_{i} L_{n}\right)+\frac{c_{4}}{4} \operatorname{Tr}\left(\left[L_{k}, T_{i}\right]\left[L_{k}, L_{n}\right]\right)\right. \\
& \left.-\frac{c_{6}}{8} \operatorname{Tr}\left(T_{i}\left[L_{k}, L_{l}\right]\right) \operatorname{Tr}\left(L_{n}\left[L_{k}, L_{l}\right]\right)\right)
\end{aligned}
$$

and they enter the kinetic energy of the Lagrangian (11) as

$$
\mathcal{T}=\frac{1}{2} a_{i} U_{i j} a_{j}-a_{i} W_{i j} b_{j}+\frac{1}{2} b_{i} V_{i j} b_{j},
$$

where the isospin and spin angular momenta, respectively, are defined as

$$
a_{i} \equiv-i \operatorname{Tr}\left[\tau_{i} A^{-1} \dot{A}\right], \quad b_{i} \equiv i \operatorname{Tr}\left[\tau_{i} \dot{B} B^{-1}\right],
$$

and they act on the static Skyrme field, $U_{0}\left(x^{i}\right)$, as

$$
U=A U_{0}\left(R_{j}^{i} x^{j}\right) A^{-1}, \quad R_{j}^{i}=\operatorname{Tr}\left[\tau^{i} B \tau_{j} B^{-1}\right],
$$

where $B(t)(A(t))$ is an $\mathrm{SU}(2)$ matrix that transforms the static Skyrmion to the (iso)spinning Skyrmion.

\section{B. Symmetry}

One could contemplate how to extract the symmetries from a numerical Skyrmion configuration. One guess could be to use the tensors of inertia that encode geometrical information about the soliton, in particular, related to its spinning and isospinning. Another more brute-force attempt could be to take moments of the energy, schematically $\int\left(x^{1}\right)^{n_{1}}\left(x^{2}\right)^{n_{2}}\left(x^{3}\right)^{n_{3}} \mathcal{E}$. This, in principle, could extract further geometrical information from the numerical Skyrmion.

However, we are interested in a particular symmetry, namely, octahedral [39] symmetry, and would like to know when it is broken to its tetrahedral subgroup. Therefore, we can use the transformations of the octahedral symmetry that are not symmetry transformations of the tetrahedral subgroup. In the following, we place a cube such that the Cartesian axes are perpendicular to three of its faces and the origin is at the center of the cube. The tetrahedral symmetry group contains the following transformations: the identity, three $C_{2}$ transformations, and eight $C_{3}$ transformations. The $C_{2}$ transformations rotate the cube by $\pi$ around one of the Cartesian axes, and the $C_{3}$ transformations rotate the cube by $\pm 2 \pi / 3$ around an axis in the $(1,1,1),(-1,-1,1)$, $(1,-1,-1)$, or $(-1,1,-1)$ direction. The octahedral symmetry group includes another six $C_{2}$ transformations as well as three $C_{4}$ transformations. The $C_{2}$ transformations rotate the cube by $\pi$ in the $(1,1,0),(-1,1,0),(1,0,1)$, $(1,0,-1),(0,-1,1)$, or $(0,-1,-1)$ direction, and the $C_{4}$ transformations rotate the cube by $\pi / 2$ around one of the Cartesian axes. We should choose a transformation among the latter two, which only resides in the octahedral symmetry group and is lost when only a tetrahedral subgroup of the symmetry is preserved.

We choose to construct an order parameter for the octahedral symmetry as follows. Let us choose one of the $C_{4}$ symmetry transformations, say about the $x$ axis. The meaning of the Skyrmion possessing such a discrete symmetry is, of course, that after rotating the Skyrmion by $\pi / 2$ about the $x$ axis we must subsequently perform an appropriate rotation in isospin space to get back to the original Skyrmion. The appropriate isospin rotation to follow the (spatial) $C_{4, x}$ rotation is a rotation by $\pi$ in isospin space about the $\pi_{1}$ axis. The 4-Skyrmion possessing octahedral symmetry will be invariant under these two subsequent transformations, while the tetrahedrally symmetric 4-Skyrmion will not be. We can thus construct the order parameter for octahedral symmetry as follows. We perform the $C_{4}$ transformation as well as the $C_{2}$ transformation in isospin space on the Skyrmion and then subtract off the original Skyrmion, take a 2-norm of the resulting field, and finally integrate over space. If the symmetry is preserved, this integral vanishes. We thus define

$$
\sigma^{O_{h}} \equiv \frac{1}{V} \int d^{3} x \operatorname{Tr}\left[\left(\left(e^{i \pi \hat{I}_{1}} e^{\frac{i \pi}{2} \hat{J}_{1}}-1\right) U\right)^{\dagger}\left(e^{i \pi \hat{I}_{1}} e^{\frac{i \pi}{2} \hat{J}_{1}}-1\right) U\right],
$$


where we have divided by the volume of the Skyrmion, $V \equiv \frac{4}{3} \pi r_{4}^{3}$, in order to get a dimensionless result.

With all observables at hand, we are now ready to turn to the numerical calculations.

\section{NUMERICAL RESULTS}

The numerical calculations in this paper are all carried out on cubic lattices of size $121^{3}$ with a spatial lattice constant of about $h_{x}=0.08$, and the derivatives are approximated by a finite difference method using a fourth-order stencil. In previous works, we were able to use the relaxation method with a forward-time algorithm, but that turned out to be too slow for the generalized Skyrme model when including the BPS-Skyrme term (with nonvanishing $c_{6}$ ). In this paper, therefore, we used the method of nonlinear conjugate gradients to find the numerical solutions. Although standard implementations of the algorithm work smoothly for small values of the potential parameter $m_{2} \lesssim 1$, some nontrivial tweaking and a sophisticated line search algorithm were needed for ensuring convergence in the large- $m_{2}$ part of the parameter space. In particular, we found a viable solution based on switching between the Newton-Raphson algorithm and a line search using a quadratic fit along the search direction of the conjugate gradients method.

For a handle on the precision, we checked that the numerically integrated baryon charge was captured by the solution to within $0.15 \%$. In addition to this, we stopped the algorithm when a local precision of the equation of motion better than $1.7 \times 10^{-6}$ was obtained.

The solutions obtained and presented here are made on a square grid in parameter space with $c_{6}=0,0.1,0.2, \ldots, 1$ and $m_{2}=0,0.1,0.2, \ldots, 6$, yielding a total of 671 numerical solutions. The baryon charge density isosurfaces are

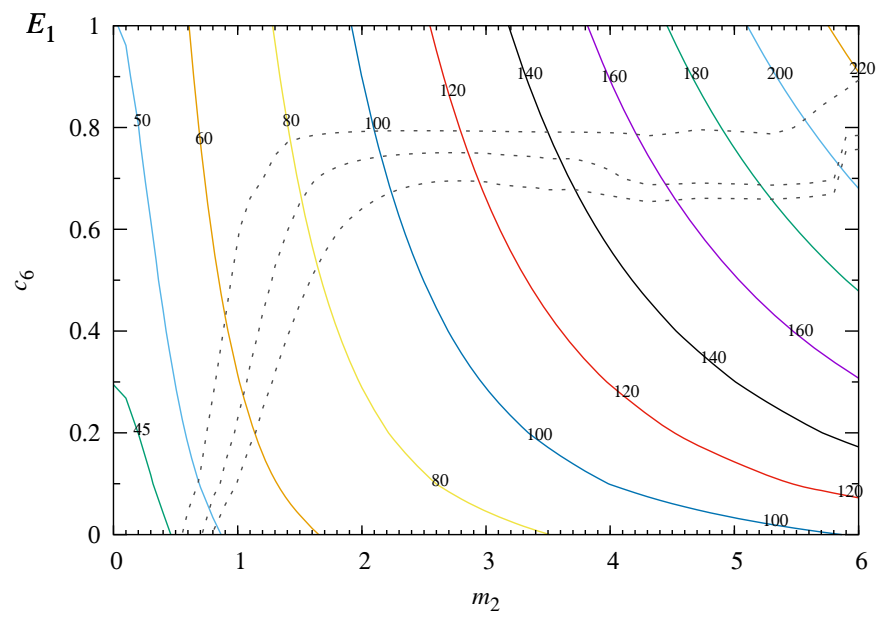

(a) shown in Figs. 11-13. As mentioned in the previous section, the initial condition for the 4-Skyrmion at the point $\left(m_{2}, c_{6}\right)=(0,0)$ is given by the rational map ansatz (19)-(21).

The figures in this section are contour plots in the parameter space with $m_{2}$ being the abscissa and $c_{6}$ being the ordinate. On all figures, we will overlay three lines with the order parameter, $\sigma^{O_{h}}$, which measures if the solutions possess octahedral symmetry $\left(\sigma^{O_{h}}=0\right)$ or remain only tetrahedrally symmetric $\left(\sigma^{O_{h}}>0\right)$. From the top of the figures and down, the curves correspond to $\sigma^{O_{h}}=0.1$, $0.5,1$.

First, we plot the classical energies (masses) of the 1-Skyrmion and the 4-Skyrmion in Skyrme units in Fig. 2 just to get a feel for how the energy changes in parameter space before calibrating the model. Both figures show isocurves according to our expectation; i.e., the energy increases roughly in quadrature from the contribution due to the loosely bound potential with coefficient $m_{2}^{2}$ and from the BPS-Skyrme term with coefficient $c_{6}$. The increase in energy, nevertheless, is quite drastic. If we compare the $\left(m_{2}, c_{6}\right)=(0,0)$ point with the $\left(m_{2}, c_{6}\right)=(6,1)$ point, the energy increases with a factor of 5.42 for the 1-Skyrmion and 5.95 for the 4-Skyrmion.

In Fig. 3, the calibration constants, i.e., the pion decay constant, $\tilde{f}_{\pi}$ and the Skyrme coupling constant $e$ are shown in the parameter space. It is interesting that for small $m_{2}<1$ the pion decay constant [see Fig. 3(a)] with our calibration convention is almost independent of $c_{6}$. What happens in this regime is that the sextic term increases both the size and the energy of the 4-Skyrmion such that the ratio is almost constant $r_{4}\left(c_{6}\right) / E_{4}\left(c_{6}\right) \propto$ const. For large $m_{2}$, however, the mass of the 4-Skyrmion increases faster than the radius, and hence the above-mentioned linear relation no longer holds; as a result, the pion decay constant

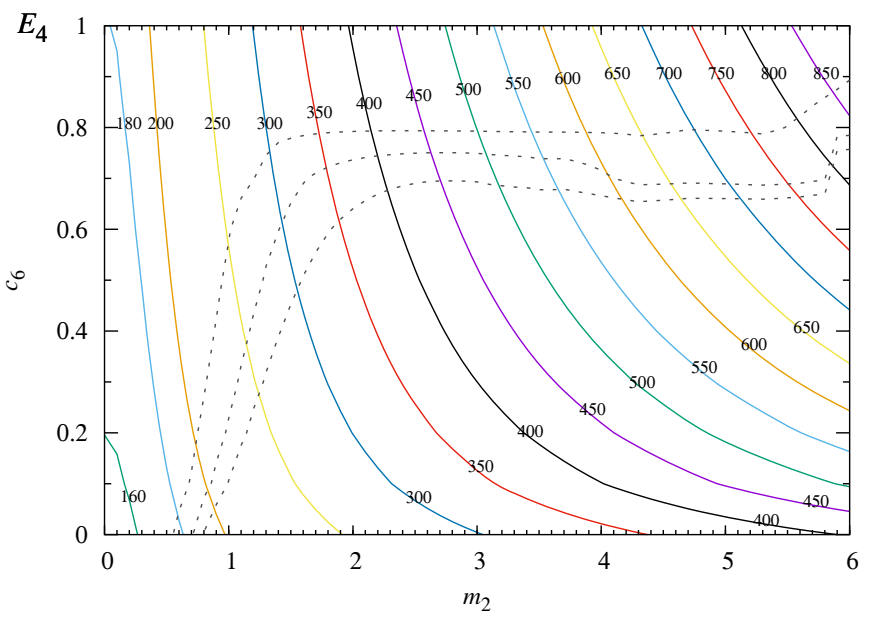

(b)

FIG. 2. Energies of the (a) 1-Skyrmion and (b) 4-Skyrmion in Skyrme units. The dashed lines show contours of $\sigma^{O_{h}}=0.1,0.5,1$ from top to bottom. 


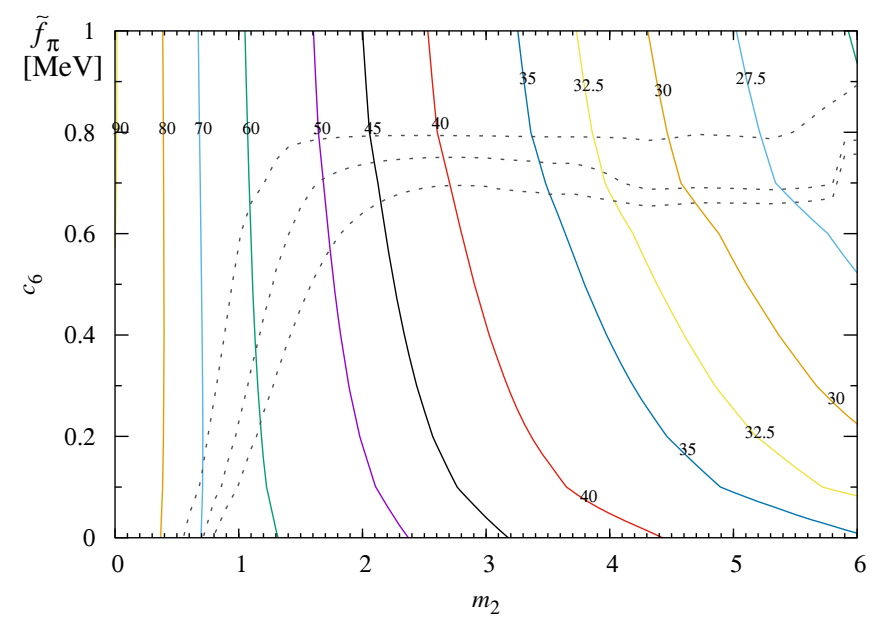

(a)

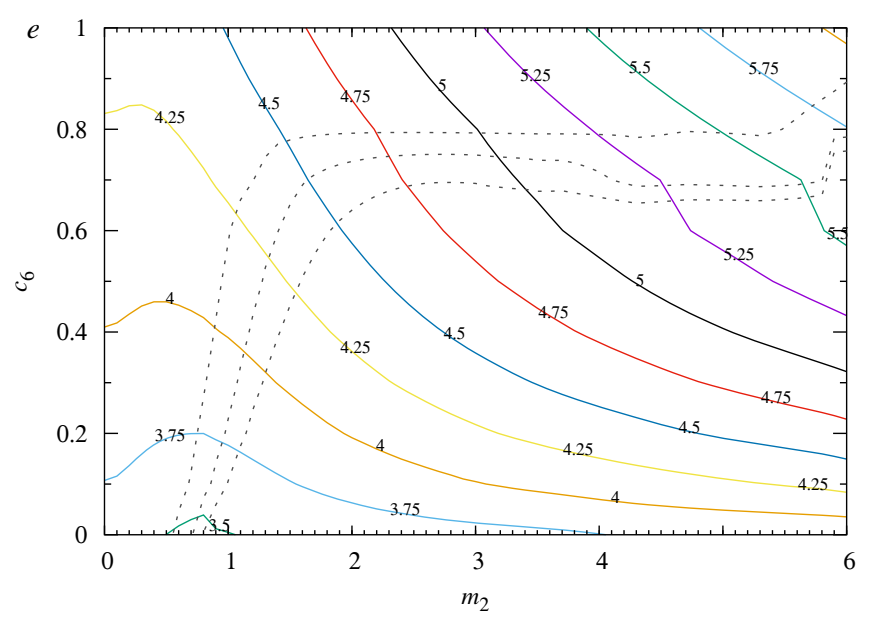

(b)

FIG. 3. Calibration constants (a) $\tilde{f}_{\pi}(\mathrm{MeV})$ and (b) the Skyrme coupling constant $e$. The dashed lines show contours of $\sigma^{O_{h}}=0.1,0.5$, 1 from top to bottom.

decreases for increasing $c_{6}$. If we now hold $c_{6}$ fixed, an increase in $m_{2}$ increases the mass and reduces the size of the Skyrmions and hence always leads to a decrease in the pion decay constant. The combined behavior is displayed in Fig. 3(a). The pion decay constant is underestimated everywhere, since in the pion vacuum its experimentally measured value is about $184 \mathrm{MeV}$.

The Skyrme coupling constant $e$ is shown in Fig. 3(b) and depends on the product of the size and the energy of the 4-Skyrmion and hence displays different behavior. For small $c_{6} \ll 1$, the increase in $m_{2}$ has a mild behavior since the loosely bound potential both increases the mass and reduces the size of the Skyrmions. For $c_{6}=0$ and $m_{2} \lesssim 0.7$, the coupling reduces slightly with increasing $m_{2}$, whereas for $m_{2}>0.7$, the coupling starts to increase. This behavior for constant slices of $c_{6}$ is continued, but the turning point ( 0.7 above) moves slightly downward as $c_{6}$ is increased. For finite $c_{6}$, the increase in $m_{2}$ now leads to a larger increase in the coupling $e$.

We will now show the spectrum of the model, starting in Fig. 4 with the nucleon mass and the $\Delta$ mass. The nucleon mass [Fig. 4(a)] in our calibration scheme is tightly related to the total binding energy (QRBE), because we fit the mass and size of the 4-Skyrmion to those of helium-4. Therefore, once the binding energy is right, then so is the nucleon mass. We will thus discuss this in more detail shortly, but let us mention that in the top-right part of the parameter space, i.e., for large $m_{2}$ and large $c_{6}$, the nucleon mass is only overestimated by about $28 \mathrm{MeV}$, which is about $3 \%$ above the experimentally measured value.

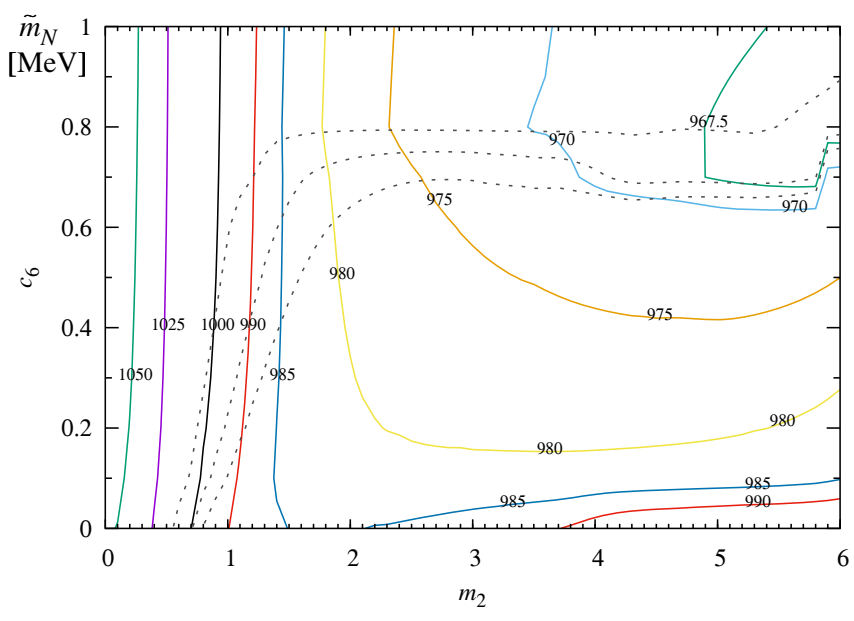

(a)

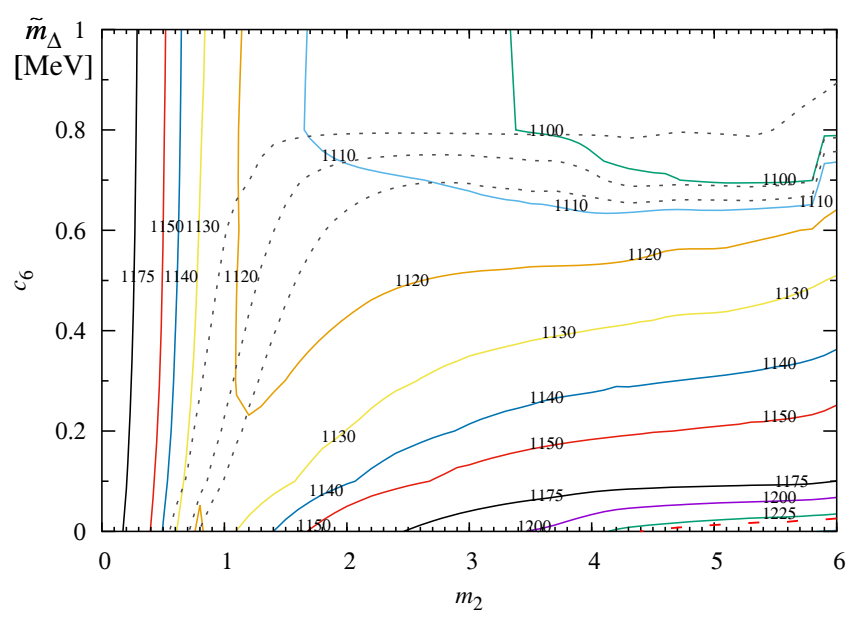

(b)

FIG. 4. (a) Nucleon mass, $\tilde{m}_{N}(\mathrm{MeV})$ and (b) $\Delta$ mass, $\tilde{m}_{\Delta}(\mathrm{MeV})$. The thick red dashed line in (b) is the experimentally measured mass of the $\Delta$ resonance $\left(\tilde{m}_{\Delta}^{\exp } \simeq 1232 \mathrm{MeV}\right)$. The dashed lines show contours of $\sigma^{O_{h}}=0.1,0.5,1$ from top to bottom. 
The $\Delta$ mass is shown in Fig. 4(b). First of all, we should warn the reader about identifying this spin excitation of the 1-Skyrmion with the $\Delta$ resonance, as it may be inherently inconsistent (see also the discussion). Nevertheless, we will show the results for completeness. As usual in a Skyrmelike model with this interpretation of the $\Delta$, its mass is underestimated. What is worse is that where the binding energy and nucleon mass tend to their experimentally measured values the $\Delta$ mass tends to be the smallest and hence farthest from its true value.

The pion mass is shown in Fig. 5(a). In our chosen calibration scheme, the dependence on $c_{6}$ is mild, and it mostly depends on the value of $m_{2}$; that is, the pion mass decreases for increasing $m_{2}$. For $m_{2} \simeq 0.5-1$, the pion mass fits well with the experimentally measured value for our choice of $m_{1}=1 / 4$. To get a more physical value of the pion mass in the top-right corner of the parameter space, we could increase the value of $m_{1}$ to say about 0.5 in order to compensate the decrease in the physical value caused by the loosely bound potential. We have not done this, as this is not a pressing issue for the model at the moment; the value of the pion decay constant is very far from its experimental value, and the QRBE is not quite at the physical values either. One attitude about the low-energy constants is that they should be "renormalized" to the inmedium conditions that the inside of the baryons possess. If this really justifies the pion decay constant to differ by more than factors of 2 (4) from its experiment value, then the same may apply to the pion mass.

The axial coupling of the nucleon is shown in Fig. 5(b). In the region of parameter space where the sextic term dominates $\left(c_{6} \sim 1\right.$ and $\left.m_{2}=0\right)$, the axial coupling is generally too large (however, this may change for larger values of $c_{6}$ than studied here), whereas for large $m_{2}$, $c_{6}=0$, the axial coupling is generally too small. In the

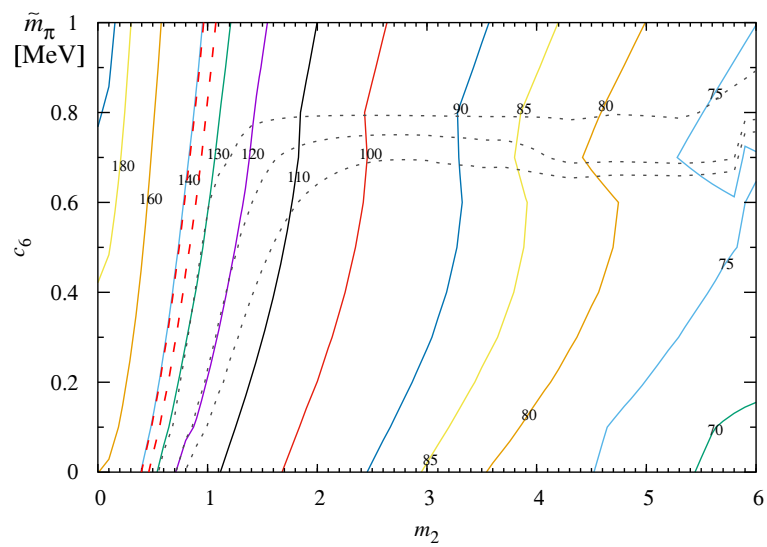

(a) top-right corner of parameter space where the binding energy turns out to be smallest, the axial coupling takes on intermediate values. Unfortunately, the experimentally measured value (thick red dashed line) is reached just after the cubic symmetry of the 4-Skyrmion is lost.

The quantum mass correction to the nucleon as a 1-Skyrmion is shown in Fig. 6(a), and the diagonal value of the isospin inertia tensor $\Lambda$ is shown in Fig. 6(b). The two quantities are related by Eq. (26). As already mentioned, there are two limits where the classical binding energy can become infinitesimally small: the point particle model limit (PPML), which corresponds to $c_{6}$ fixed, $m_{2} \rightarrow \infty$, and the BPS-Skyrme model limit (BSML), which corresponds to $c_{6} / m_{2}$ fixed, $m_{2} \rightarrow \infty$. It is interesting to see-within the calibration scheme adopted herethat the direction of the BPS-Skyrme model limit reaches quantum mass corrections to the 1-Skyrmion that are almost half the values obtained in the point particle model limit. If this strategy for obtaining a physically sensible Skyrme model is correct, it is important to see where the quantum correction to the 1-Skyrmion can be suppressed enough to reach physically measured values of the binding energies. This viewpoint is typical for a purist particle physicist who prefers as little fine-tuning as possible. Other possibilities are, of course, that the physics at the atomic scale is highly fine tuned and there are big corrections to both the 1-Skyrmions and the $B$-Skyrmions canceling each other out almost precisely, leaving behind binding energies at the $1 \%$ level (see also the discussion).

The quantity $\tilde{\epsilon}_{1}$ contains the energy unit, the value of the Skyrme coupling, and the isospin inertia tensor's diagonal value. To disentangle the various effects, we show the value of the diagonal of the isospin inertia tensor, $\Lambda$, in Fig. 6(b). As $\tilde{\epsilon}_{1} \propto \Lambda^{-1}$, we can see that, overall, the above-mentioned behavior indeed stems from $\Lambda$ and not peculiarities of the

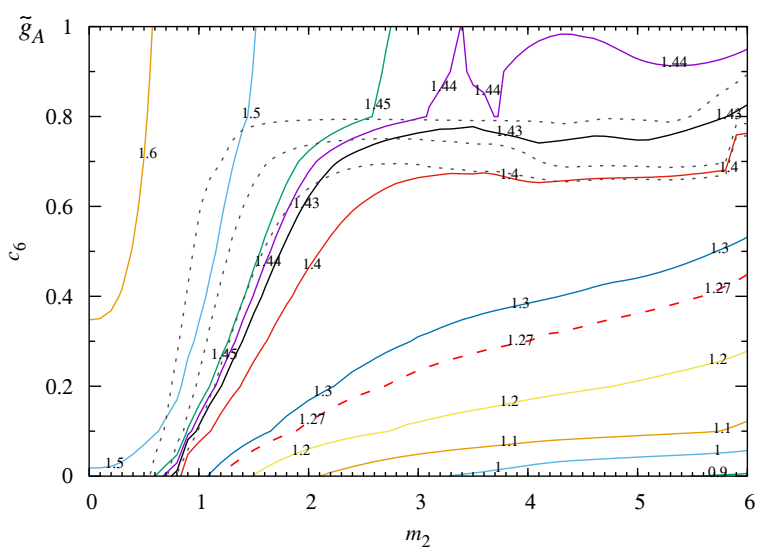

(b)

FIG. 5. (a) pion mass, $\tilde{m}_{\pi}(\mathrm{MeV})$ and (b) axial coupling $\tilde{g}_{A}$. The thick red dashed lines in (a) are the experimentally measured pion masses $\left(\tilde{m}_{\pi}^{\exp } \simeq 135.0 \mathrm{MeV}, 139.6 \mathrm{MeV}\right)$ and in (b) are the experimentally measured axial coupling of the nucleon $\left(\tilde{g}_{A}^{\exp } \simeq 1.27\right)$ [40]. The dashed lines show contours of $\sigma^{O_{h}}=0.1,0.5,1$ from top to bottom. 


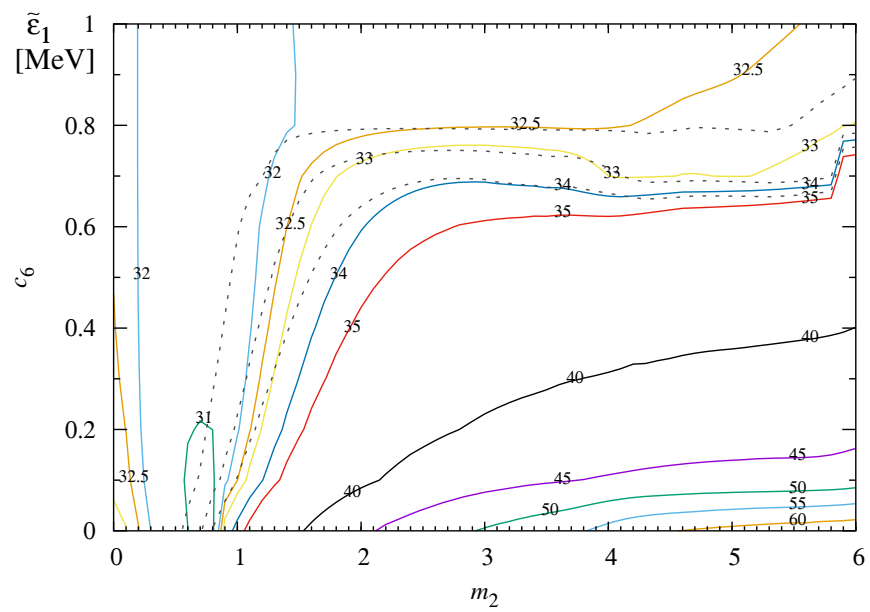

(a)

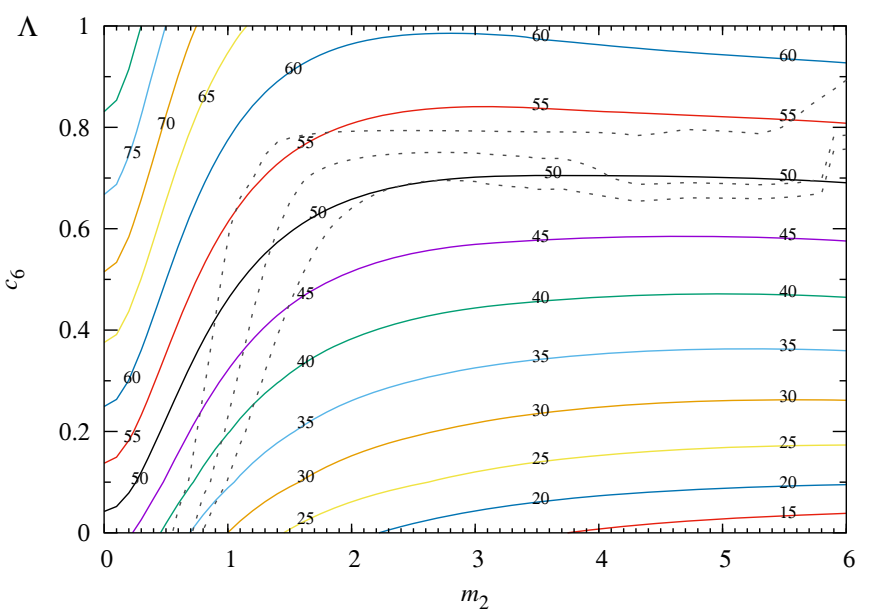

(b)

FIG. 6. (a) Quantum isospin correction to the nucleon mass, $\tilde{\epsilon}_{1}(\mathrm{MeV})$, and (b) the diagonal of the isospin inertia tensor of the 1-Skyrmion, $\Lambda$, in Skyrme units. The dashed lines show contours of $\sigma^{O_{h}}=0.1,0.5,1$ from top to bottom.

calibration. That is, the smallness of the quantum correction in the BPS-Skyrme model limit comes from the fact that $\Lambda$ is bigger than in the point particle model limit. This fact, in turn, can be traced directly to the fact that the Skyrmions become larger when a sizable sextic term (BPS-Skyrme term) is included and they become smaller when a strong loosely bound potential is turned on.

We are now ready to present one of the main results of the paper, namely, the relative binding energies in Fig. 7. In this part of parameter space, the CRBE [Fig. 7(a)] is almost independent of $c_{6}$. That is, only cranking up the sextic term does not reduce the CRBE, but in fact-in this calibration scheme-it leads to a slight increase in the binding energy.
The effect is quite mild, though. The explanation is that the sextic term just makes the Skyrmions larger and heavier; however, after the calibration, this effect is almost swallowed up. The loosely bound potential, on the other hand, does its job very well. The CRBE is reduced to the $1 \%$ level around $m_{2} \sim 2-2.6$ depending on the value of $c_{6}$, and it reaches values slightly below $0.2 \%$ for $m_{2} \sim 6$. Now, the sextic term is crucial for what happens. If we solely include the loosely bound potential, the model loses the platonic symmetries of the Skyrmions, and the Skyrmions become well-separated point particles. However, if we turn on the BPS-Skyrme term, the 4-Skyrmion can retain its cubic symmetry and possess a CRBE below the $1 \%$ level. The

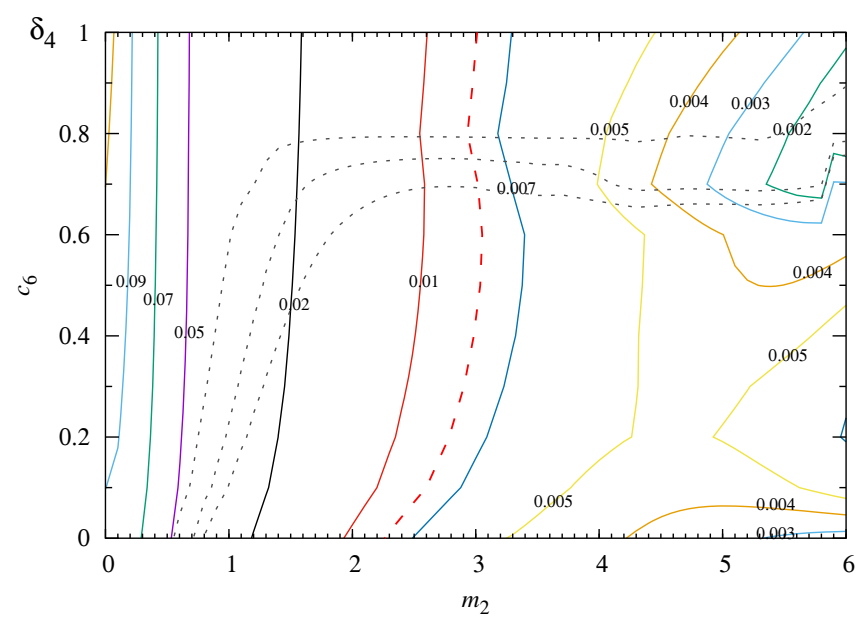

(a)

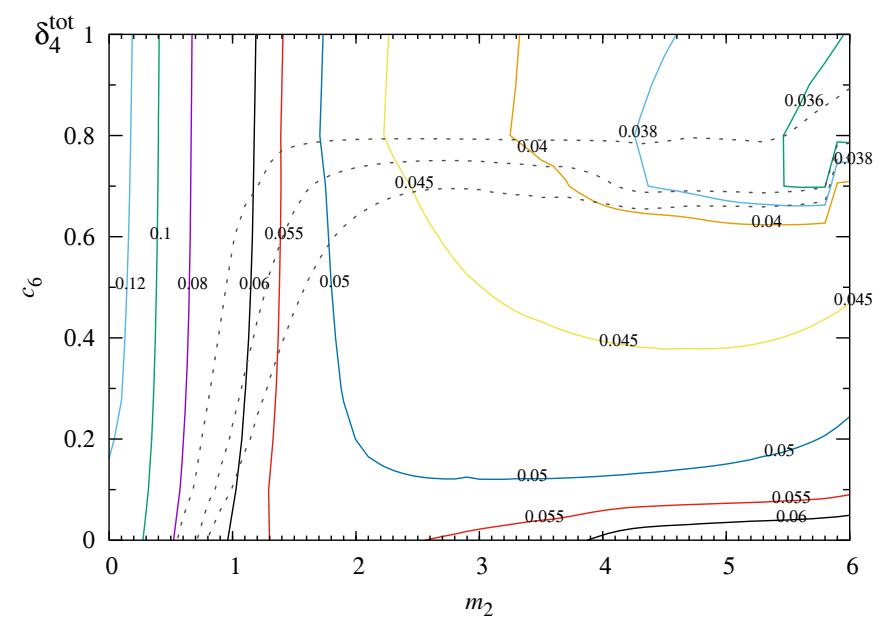

(b)

FIG. 7. (a) Classical and (b) quantum relative binding energy of the 4-Skyrmion. The thick red dashed line in (a) is the experimental binding energy of helium-4 ( $\delta_{4}^{\exp } \simeq 0.008$ ) (the thick red dashed line should not be compared to the CRBE but to the QRBE; it is shown just for reference). The dashed lines show contours of $\sigma^{O_{h}}=0.1,0.5,1$ from top to bottom. 
upper black dashed line shows the symmetry order parameter for $\sigma^{O_{h}}=0.1$, and the cubic symmetry can also be observed in Figs. 11-13.

The problem of the binding energies has not quite been solved yet, because we have to identify the quantum state of the Skyrmion with the nuclear particle. This, in particular, means that all binding energies are relatively increased by the fact that the nucleon receives a quantum correction for being a spin- $\frac{1}{2}$ particle in the ground state. This means that if it is a consistent treatment not to include any other quantum corrections (which is probably not the case) then we must find a point in the parameter space where the quantum mass correction to the 1-Skyrmion is at the $1 \%$ level. In Fig. 7(b), we can see that the discussion of the quantum correction $\tilde{\epsilon}_{1}$ carries directly over to the QRBE. In particular, in the direction of the point particle model limit $\left(c_{6}=0, m_{2} \rightarrow \infty\right)$, the QRBE we reach in the parameter space is only as low as slightly below $6 \%$, whereas in the direction of the BPS-Skyrme model limit $\left(c_{6} \propto m_{2} \rightarrow \infty\right)$, the QRBE reaches values as low as 3.6\%, which for helium-4 should be compared to about $0.8 \%$ (experimental value), i.e., about $2.8 \%$ overbinding. This is, however, the level of overbinding also sometimes present in nuclear models like the $a b$ initio no-core shell model; see, e.g., Ref. [41].

We will now turn to the electric charge radius of the proton, which is shown in Fig. 8(a). In the Skyrme model, it has half its contribution from the baryon charge density and the other half from the vector charge corresponding to the isospin; see Eq. (29). It is interesting to see that we can obtain the best QRBE for large $m_{2}$ and large $c_{6}$ and hence also the best nucleon mass, but the physically measured value of the electric charge radius of the proton is reached spot on in the direction of the point particle model limit, that is, for $c_{6}=0$ and $m_{2} \sim 5-6$. In all other parts of parameter space, the 1-Skyrmion size is generally overestimated. This is due to the fact that $B$-Skyrmions tend to be too small and the 4-Skyrmion is no exception. Because of the calibration in which we fit the size of the 4-Skyrmion to that of helium-4, the 1-Skyrmion is thus generally too large. It is interesting, nevertheless, to see that the point particle model limit gets the proton size right.

The baryon charge radius is not physically measurable, but it is a component of the electric charge radius of the proton. For large $m_{2}$, we can see that their behaviors are comparable; see Fig. 8(b).

The last but important observable we will study here is our proposal for an order parameter for the symmetry breaking of the cubic (octahedral) symmetry of the 4Skyrmion; see Eq. (39). By comparing the symmetries observed in Figs. 11-13 with the values seen in Fig. 9(a), we see that for $\sigma^{O_{h}}<0.1$ the 4-Skyrmion possesses cubic symmetry, which is the upper black dashed line shown on all figures. Let us mention that in the region of parameter space to the top-left of the upper black dashed line $\sigma^{O_{h}}$ is very close to zero everywhere, except close to the dashed line, and the deviation here is merely numerical error. For $\sigma^{O_{h}} \gtrsim 0.5$, the loss of octahedral symmetry is visible to the naked eye; see Figs. 11-13.

For completeness, we display the values of the inertia tensors in the parameter space in Figs. 9(b) and 10. Throughout the scanned part of the parameter space, we have that $V_{i j}=v \delta^{i j}$ is diagonal and $W_{i j}=0$ vanishes, whereas the two nonzero values of the isospin tensor of inertia are $U_{11}=U_{22}$ and $U_{33}$. We can see from Fig. 10 that $U_{11}$ is in general different from $U_{33}$ except in the region of parameter space where $c_{6}$ is small and $m_{2}$ is large.

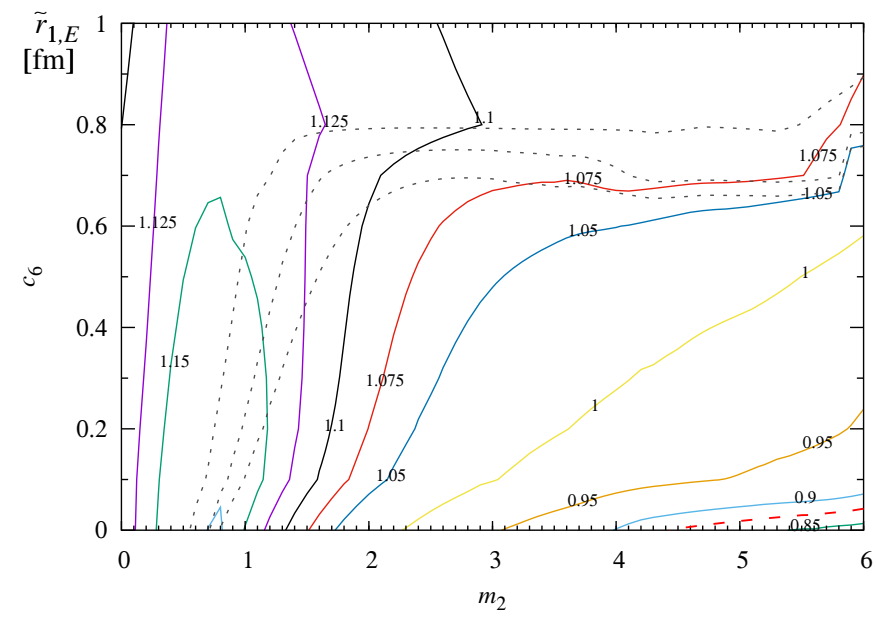

(a)

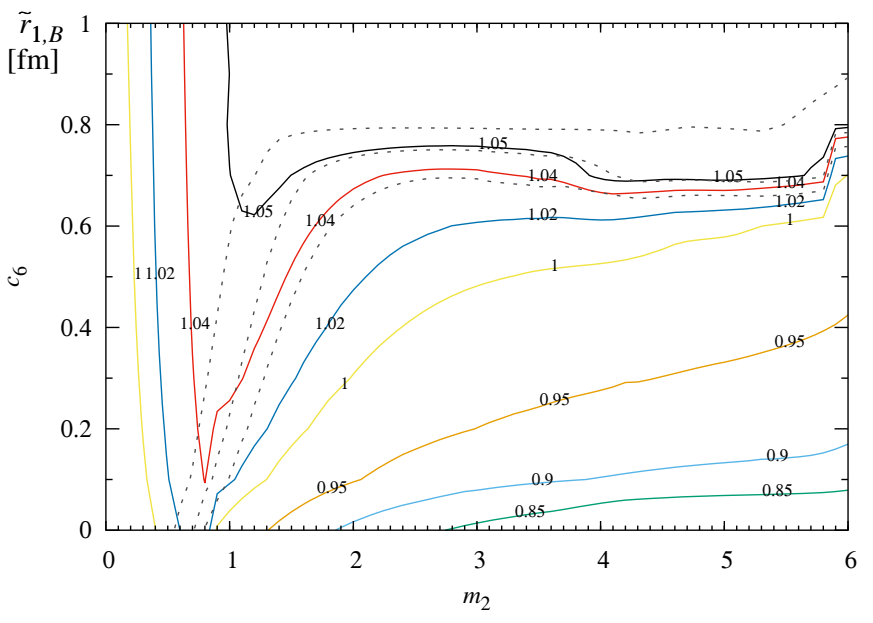

(b)

FIG. 8. (a) $\left(\tilde{r}_{1, E}\right)$ Electric and (b) $\left(\tilde{r}_{1, B}\right)$ baryon charge radii of the proton. The thick red dashed line in (a) is the experimentally measured charge radius of the proton (CODATA) $\left(\tilde{r}_{1, E}^{\exp } \simeq 0.875 \mathrm{fm}\right)[40]$. The dashed lines show contours of $\sigma^{O_{h}}=0.1,0.5,1$ from top to bottom. 


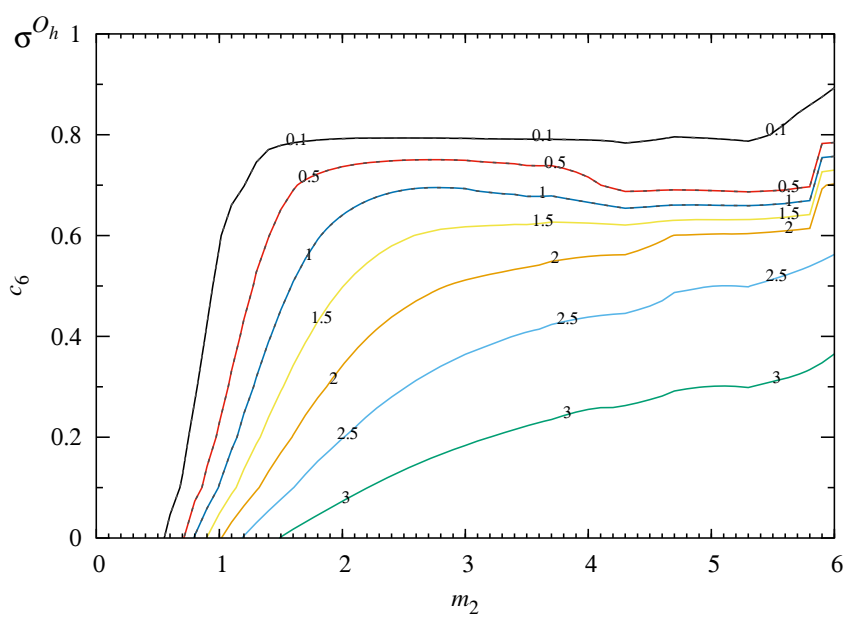

(a)

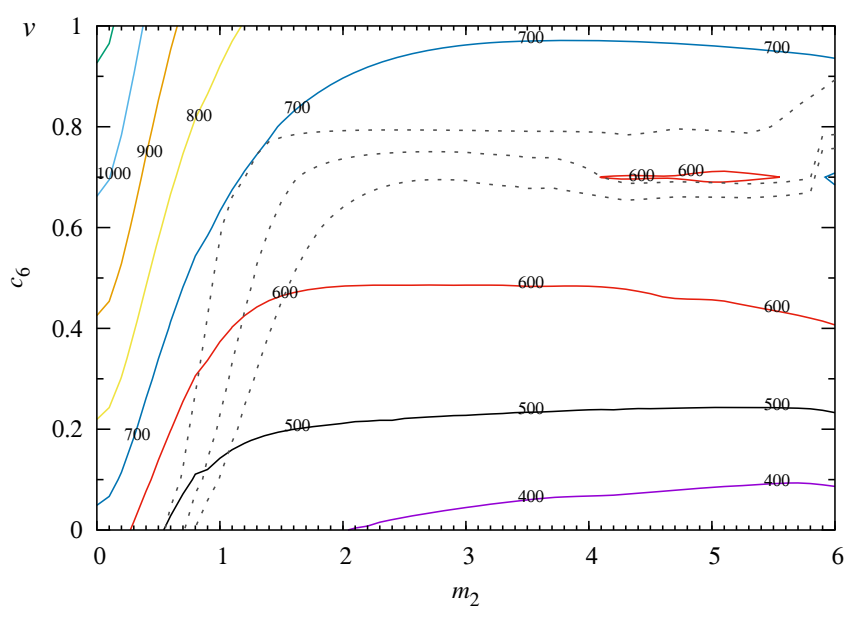

(b)

FIG. 9. (a) Order parameter for octahedral symmetry $\sigma^{O_{h}}$ and (b) diagonal component $v$ of the spin inertia tensor of the 4-Skyrmion, $V_{i j}=v \delta^{i j}$, in Skyrme units. The dashed lines show contours of $\sigma^{O_{h}}=0.1,0.5,1$ from top to bottom.

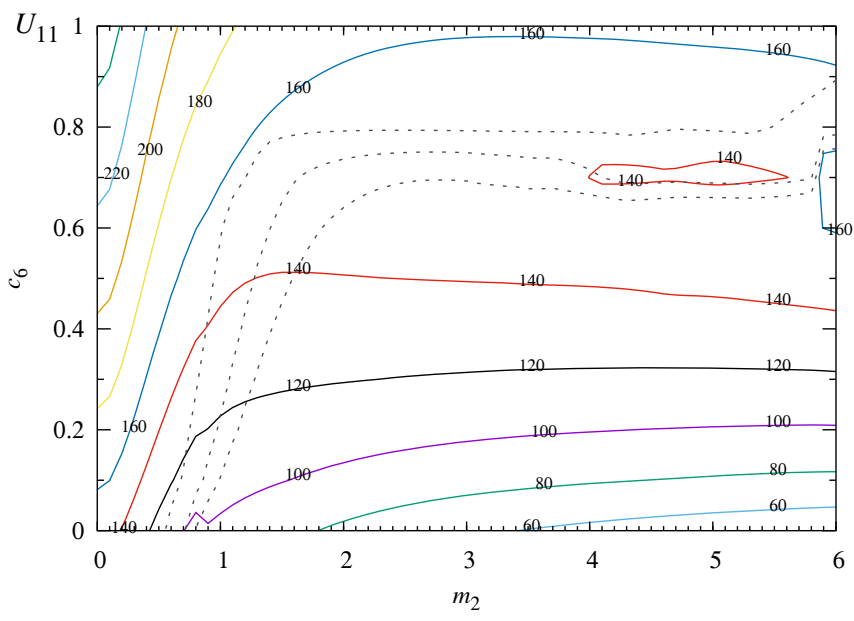

(a)

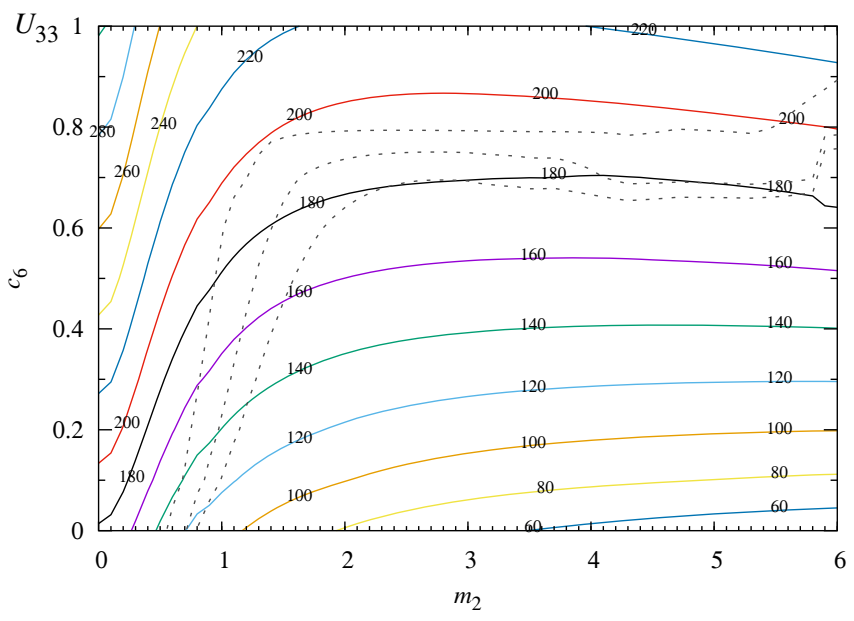

(b)

FIG. 10. (a) $U_{11}=U_{22}$ and (b) $U_{33}$ of the isospin inertia tensor of the 4-Skyrmion in Skyrme units. The dashed lines show contours of $\sigma^{O_{h}}=0.1,0.5,1$ from top to bottom.

\section{DISCUSSION}

In this paper, we have made an extensive parameter scan of the generalized Skyrme model with the loosely bound potential by performing full PDE calculations. The full numerical calculations are necessary for detecting the symmetries of the Skyrmions, in particular, whether the 4-Skyrmion possesses the sought-for cubic symmetry or it loses it, becoming a tetrahedrally symmetric object of "point" particles. In Ref. [27], we made an initial study of the parameter space limited to $m_{2} \leq 1$ using the rational map ansatz for the cube, under the assumption that the symmetries would stay unchanged in such a limited part of parameter space. That assumption turned out to be true indeed. In this paper, we have been able to go much further into the direction of turning up the loosely bound potential and hence reducing the binding energies significantly. As expected from Ref. [19], the loosely bound potential itself will quickly break the cubic symmetry of the 4-Skyrmion. Fortunately, it turns out that turning on a finite sextic term makes the 4-Skyrmion resistant to the impending symmetry breaking a long way up in $m_{2}$, the (square root of the) coefficient of the loosely bound potential.

The philosophy that we have used as a guiding principle is to keep as much symmetry as possible while reducing the binding energies as much as possible; we try to cling to the platonic symmetries possessed by the Skyrmions of small $B(B<8)$ and, in particular, the cubic symmetry of the 4-Skyrmion. Next, we note that the CRBE is almost independent of the sextic term with coefficient $c_{6}$ but 


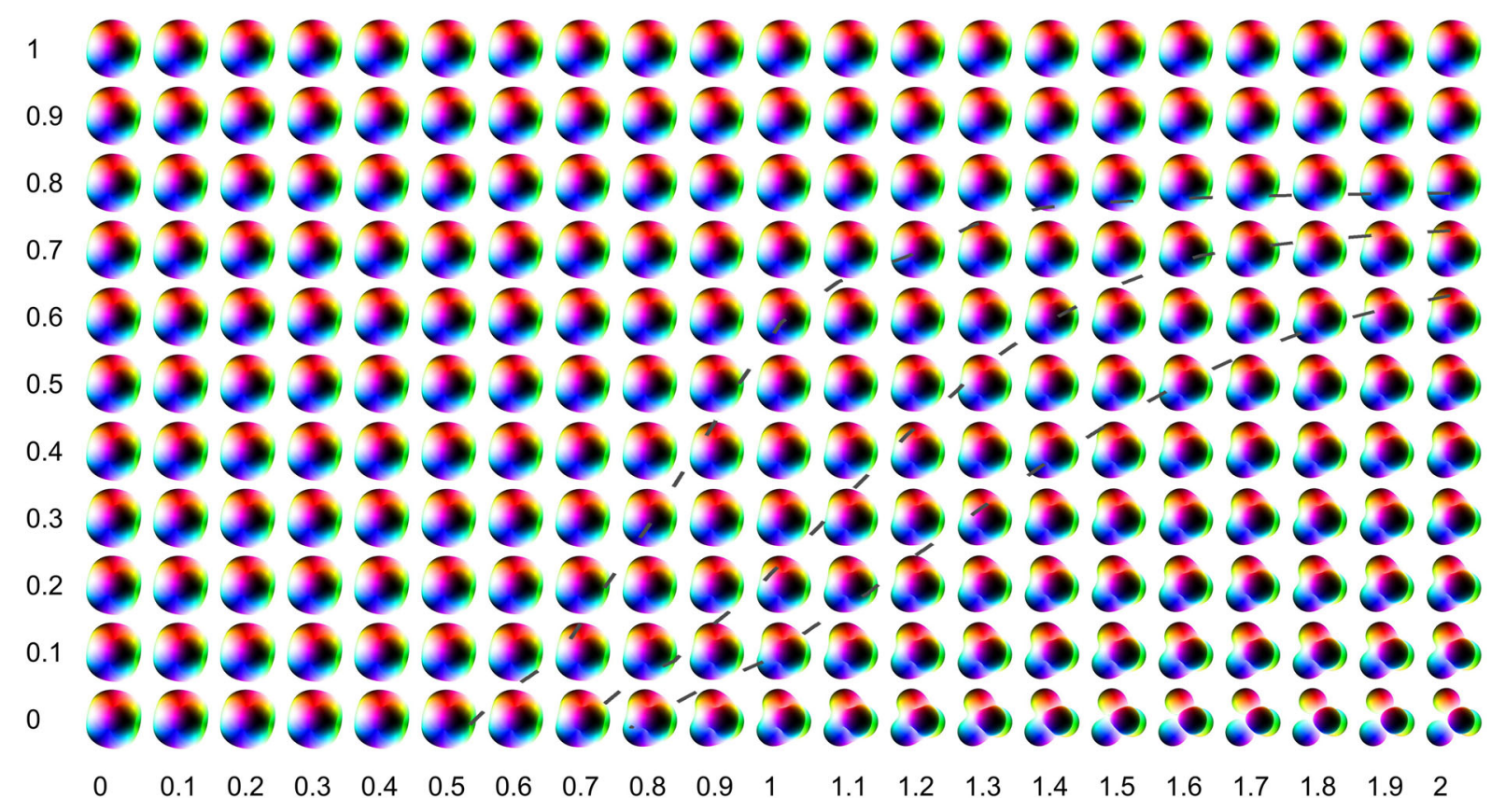

FIG. 11. Baryon charge density isosurfaces of the 4-Skyrmion solutions over part of the scanned parameter space. The vertical axis denotes the values of $c_{6}$, while the horizontal axis is $m_{2}$. The dashed lines show contours of $\sigma^{O_{h}}=0.1,0.5,1$ from top to bottom. The center of mass of the Skyrmion corresponds to its position in parameter space.

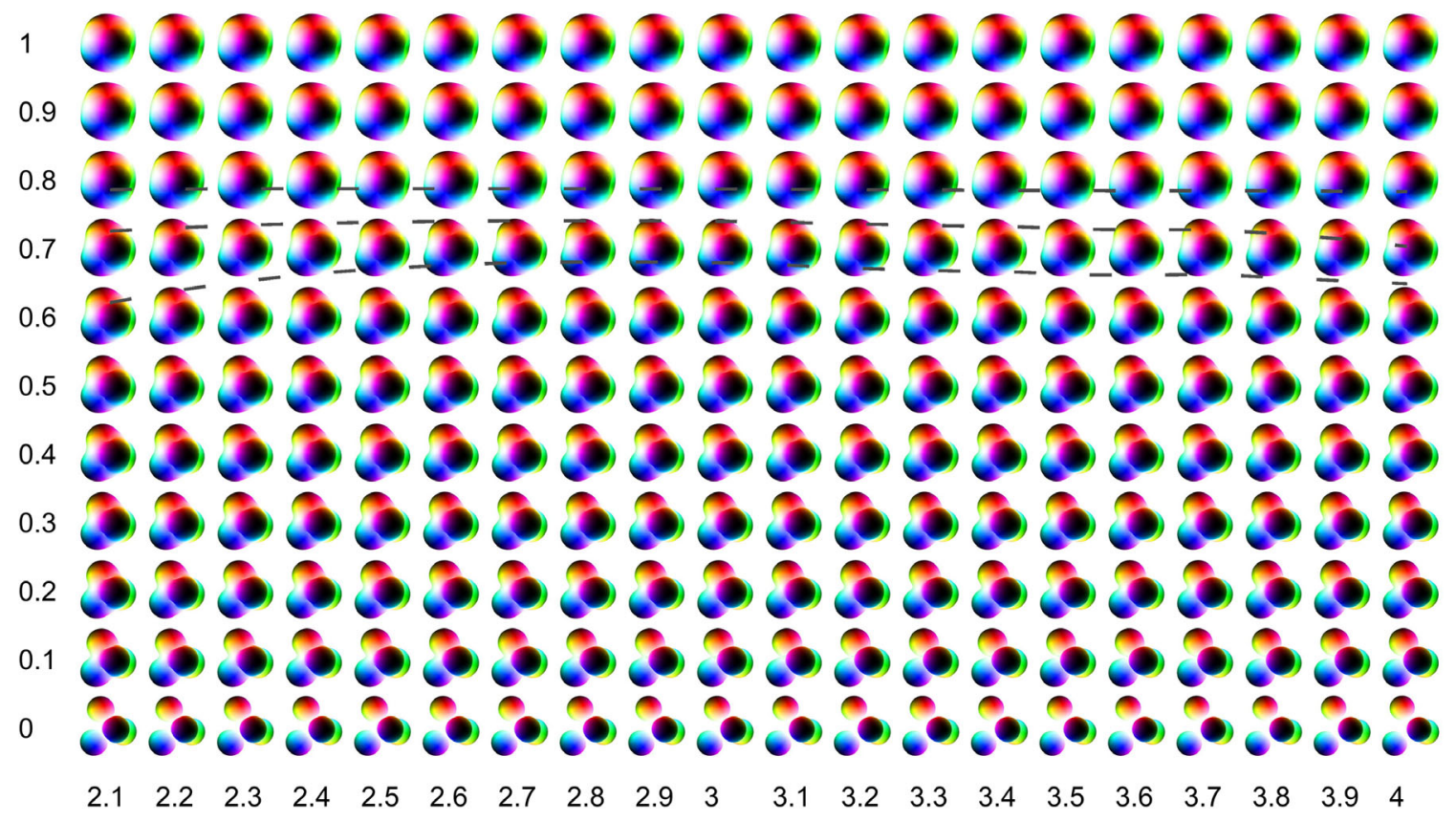

FIG. 12. Baryon charge density isosurfaces of the 4-Skyrmion solutions over part of the scanned parameter space. The vertical axis denotes the values of $c_{6}$, while the horizontal axis is $m_{2}$. The dashed lines show contours of $\sigma^{O_{h}}=0.1,0.5,1$ from top to bottom. The center of mass of the Skyrmion corresponds to its position in parameter space.

depends strongly on the loosely bound potential with coefficient $m_{2}^{2}$. Now, as a direct test for whether the above-stated philosophy is justified experimentally, we can compare two limits: the PPML $\left(c_{6}=0\right.$ and $\left.m_{2} \rightarrow \infty\right)$ and the BSML $\left(c_{6} \propto m_{2} \rightarrow \infty\right)$. Of course, we have not taken any strict limit and just considered $m_{2}$ large $\left(m_{2} \simeq 6\right)$, and the BSML case here will refer to $c_{6}=1$, $m_{2}=6$. First of all, we find that, although the CRBE is almost the same in the two cases, the QRBE receives a larger quantum contribution in the PPML case than in the BSML case. This fact is intimately related to the value of the isospin inertia tensor being larger when the sextic term 


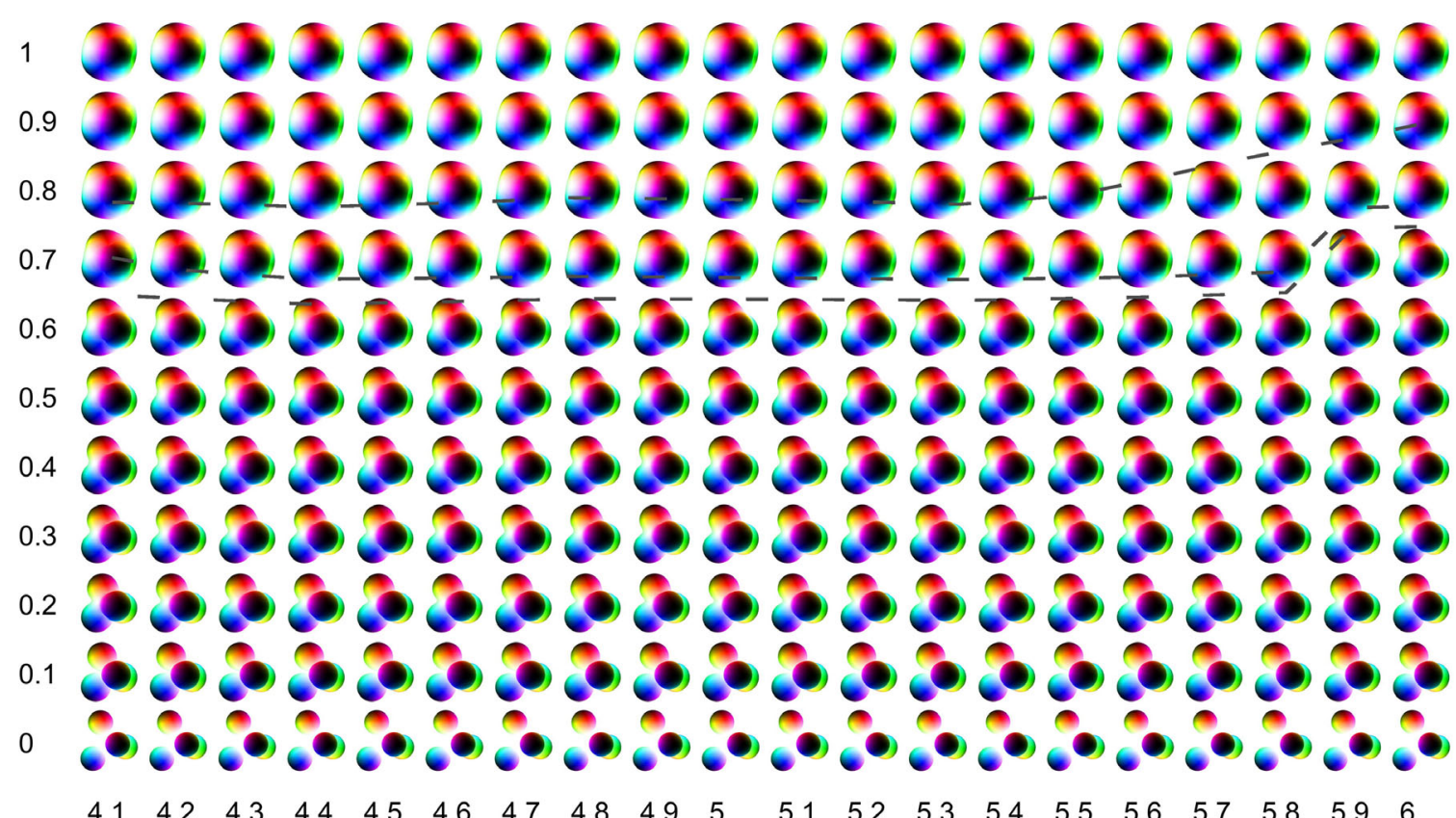

FIG. 13. Baryon charge density isosurfaces of the 4-Skyrmion solutions over part of the scanned parameter space. The vertical axis denotes the values of $c_{6}$, while the horizontal axis is $m_{2}$. The dashed lines show contours of $\sigma^{O_{h}}=0.1,0.5,1$ from top to bottom. The center of mass of the Skyrmion corresponds to its position in parameter space.

is turned on, which in turn is due to the sextic term enlarging the Skyrmions. The nucleon mass correlates with the binding energy in our calibration scheme and hence is closer to the measured value in the BSML case (but still overestimated) than in the PPML case. On the other hand, the pion decay constant is larger (but still underestimated) in the PPML case than in the BSML case, and the electric charge radius is almost spot on the experimental value in the PPML corner of our parameter space. Finally, there is a tie between the two cases for the axial coupling of the nucleon, for which it is overestimated in the BSML case and underestimated in the PPML case. We present in Table I four benchmark points compared to experimental data, including the PPML and BSML cases.
For the observables considered in this paper, it is not clear that preserving as much symmetry as possible is better in line with phenomenology. Both the cases summarized above have their merits. Nevertheless, once the full quantum excitational spectrum is considered for the nuclei, it becomes clear that having a large symmetry is not just aesthetics but a necessity. This was pointed out recently in Ref. [30]; in this particular case, the lack of a symmetry of rank 4 resulted in parity doubling of the states-not observed in nature.

A completely different way of trying to solve the binding energy problem of the Skyrmions is to disregard the classical energies completely and believe that the true quantum states have very large corrections to their classical counterparts. This solution may well be the true description

TABLE I. Benchmark points compared to experimental data. The model points are A [standard Skyrme model (SSM)], B [generalized Skyrme model (GSM)], C (PPML), and D (BSML).

\begin{tabular}{lcccc}
\hline \hline & Point A (SSM) & Point B $(\mathrm{GSM})$ & Point C (PPML) & Point D (BSML) \\
\cline { 2 - 5 } & $\left(m_{2}, c_{6}\right)=(0,0)$ & $\left(m_{2}, c_{6}\right)=(0,1)$ & $\left(m_{2}, c_{6}\right)=(6,0)$ & $\left(m_{2}, c_{6}\right)=(6,1)$ \\
\hline$\tilde{f}_{\pi}$ & $-32.1 \%$ & $-51.0 \%$ & $-80.8 \%$ & $-86.5 \%$ \\
$\tilde{m}_{N}$ & $+12.0 \%$ & $+13.9 \%$ & $+6.2 \%$ & $+2.9 \%$ \\
$\tilde{m}_{\Delta}$ & $-3.8 \%$ & $-2.6 \%$ & $+1.6 \%$ & $-11.0 \%$ \\
$\tilde{m}_{\pi}^{ \pm}$ & $+14.7 \%$ & $+39.9 \%$ & $-51.7 \%$ & $-46.2 \%$ \\
$\tilde{m}_{\pi}^{0}$ & $+18.6 \%$ & $+44.7 \%$ & $-50.1 \%$ & $-44.4 \%$ \\
$\tilde{g}_{A}$ & $+17.4 \%$ & $+30.3 \%$ & $-30.0 \%$ & $+13.6 \%$ \\
$\delta_{4}$ & $+970.3 \%$ & $+1174.5 \%$ & $-67.7 \%$ & $-72.5 \%$ \\
$\delta_{4}^{\text {tot }}$ & $+1331.4 \%$ & $+1514.6 \%$ & $+725.7 \%$ & $+348.9 \%$ \\
$\tilde{r}_{1, E}$ & $+27.8 \%$ & $+25.3 \%$ & $-4.2 \%$ & $+23.0 \%$ \\
\hline \hline
\end{tabular}


of nuclear physics, although it runs against conventional particle physics wisdom that prefers natural mechanisms as explanations for physical effects. A known counterexample for naturalness indeed in nuclear physics is the fact that the binding energy of the triplet deuteron is about $2.2 \mathrm{MeV}$, whereas the energy released in neutron beta decays is about 1.3 MeV. That $0.9 \mathrm{MeV}$ difference is what kept all the neutrons from decaying during the evolution of the Universe and be missing in the formation of countless elements. Of course, our Universe may just well be a giant accident. As we discussed in the Introduction, the question of whether semiclassical quantization with the perturbative addition of a few light modes of the soliton is a good approximation comes down to whether the fluctuation spectrum is "weakly coupled." It would be an important next step to investigate this issue in depth.

The $\Delta$ resonance is at best problematic in the Skyrme model. The reason for this is evident from our discussion about trying to reduce the quantum isospin contribution to the mass of the nucleon and is rooted in Eq. (28). That is, if we make $\tilde{\epsilon}_{1}$ small, then so is $5 \tilde{\epsilon}_{1}$; see Ref. [42]. More concretely, if we want the spin/isospin contribution to the mass of the nucleon, $\tilde{\epsilon}_{1}$, to be less than the binding energy of roughly $16 \mathrm{MeV}$, which is approximately the binding energy of nuclear matter, then it is impossible for $5 \tilde{\epsilon}_{1}$ to be as large as $366 \mathrm{MeV}[42,43]$. As further pointed out in Ref. [42], the $\Delta$ resonance probably needs a fully relativistic treatment and should be considered as a resonance with a complex mass pole.

Considering larger coefficients of the sextic BPSSkyrme term is a natural continuation of this work, and it may show qualitatively interesting new behavior of the model. If the BPS-Skyrme term and the potential term become too large, however, one enters the near-BPS regime of the BPS-Skyrme model, which is known to be technically difficult. In the light of the discussions in this paper, the more important question is whether it is necessary to obtain solutions with very low classical binding energieslike in this paper-or the true solution to the quantum physics of nuclei lies in sizable corrections that perhaps via beautiful symmetries somehow all balance in such a way as to give small binding energies at the $1 \%$ level for all nuclei. This will be left as work for the future.

We should remind the reader that we did not get the physical pion mass right in the region of parameter space with small binding energies. This can easily be fixed, but the change for the rest of the physics is expected to be insignificant, and as long as the pion decay constant is so far from its measured value, it remains a question whether the pion mass should be close to its experimental value or not. Lattice-QCD simulations often get good results even though their pion mass is typically much too large compared with the experimental value.

There are lots of directions to consider for improving the Skyrme model in order for it to become a full-fledged high-precision model of nuclear physics. If the program succeeds, it will become a few-parameter model, which basically can cover all nuclei. The list of problems is, however, not so short. The problem of the binding energy that we have worked on in this paper is not solved yet, and we are probably getting closer to a point at which we can determine whether the Skyrme model is natural, and hence the quantum corrections are somehow small as expected in systems with semiclassical quantization, or there are relatively large quantum corrections that just happen to balance out almost perfectly over a large variety of nucleimany possessing different symmetries.

The small isospin breaking present in nature still remains largely unincorporated in the Skyrme model. The recent suggestion by Speight [44] is based on including the $\omega$ meson and an explicit symmetry-breaking term. This direction of improving the Skyrme model is also considered for solving the binding energy problem, that is, including vector mesons in the model; see, e.g., Refs. [14] in which $\rho$ mesons are considered.

A further improvement to be considered, which will become more important for the studies of large nuclei, is to include the effects of the Coulomb energy. Although how to calculate the Coulomb force for multi-Skyrmions is known, it should ideally be backreacted onto the Skyrmions. This would require some partial gauging and further complicate the model.

It would also be interesting to consider Skyrmions other than the 4-Skyrmion, in order to check our claims about the preservation of symmetries in the model. A preliminary study suggests that for the 8-Skyrmion the two cubes retain their separate octahedral symmetries but become more weakly bound to each other with the result that, in the low-binding energy regime, the chain and twisted chain become almost degenerate in energies. There are plenty of other Skyrmions that would be interesting to study.

The following question, however, remains: how can we resolve the quantum part of the binding-energy problem for Skyrmions as nuclei? Hopefully, this question will be answered in the future.

\section{ACKNOWLEDGMENTS}

S. B. G. thanks Chris Halcrow for discussions. S. B. G. is supported by the Ministry of Education, Culture, Sports, Science (MEXT)-Supported Program for the Strategic Research Foundation at Private Universities "Topological Science" (Grant No. S1511006) and by a Grant-in-Aid for Scientific Research on Innovative Areas "Topological Materials Science" (KAKENHI Grant No. 15H05855) from MEXT, Japan. The calculations in this work were carried out using the Topological Science Cluster (TSC) computing cluster of the "Topological Science" project at Keio University. 
[1] T. H. R. Skyrme, A unified field theory of mesons and baryons, Nucl. Phys. 31, 556 (1962).

[2] T. H. R. Skyrme, A nonlinear field theory, Proc. R. Soc. A 260, 127 (1961).

[3] E. Witten, Global aspects of current algebra, Nucl. Phys. B223, 422 (1983).

[4] E. Witten, Current algebra, baryons, and quark confinement, Nucl. Phys. B223, 433 (1983).

[5] E. Braaten, S. Townsend, and L. Carson, Novel structure of static multi-soliton solutions in the Skyrme model, Phys. Lett. B 235, 147 (1990).

[6] C. J. Houghton, N. S. Manton, and P. M. Sutcliffe, Rational maps, monopoles and Skyrmions, Nucl. Phys. B510, 507 (1998).

[7] R. Battye, N. S. Manton, and P. Sutcliffe, Skyrmions and the alpha-particle model of nuclei, Proc. R. Soc. A 463, 261 (2007).

[8] D. T. J. Feist, P. H. C. Lau, and N. S. Manton, Skyrmions up to baryon number 108, Phys. Rev. D 87, 085034 (2013).

[9] P. H. C. Lau and N. S. Manton, States of Carbon-12 in the Skyrme Model, Phys. Rev. Lett. 113, 232503 (2014).

[10] C. J. Halcrow, C. King, and N. S. Manton, A dynamical $\alpha$-cluster model of ${ }^{16} \mathrm{O}$, Phys. Rev. C 95, 031303 (2017).

[11] J. I. Rawlinson, An alpha particle model for Carbon-12, Nucl. Phys. A975, 122 (2018).

[12] P. Sutcliffe, Skyrmions, instantons and holography, J. High Energy Phys. 08 (2010) 019.

[13] P. Sutcliffe, Skyrmions in a truncated BPS theory, J. High Energy Phys. 04 (2011) 045.

[14] C. Naya and P. Sutcliffe, Skyrmions in models with pions and rho mesons, J. High Energy Phys. 05 (2018) 174.

[15] C. Adam, J. Sanchez-Guillen, and A. Wereszczynski, A Skyrme-type proposal for baryonic matter, Phys. Lett. B 691, 105 (2010).

[16] C. Adam, J. Sanchez-Guillen, and A. Wereszczynski, A BPS Skyrme model and baryons at large $N_{c}$, Phys. Rev. D 82, 085015 (2010).

[17] D. Harland, Topological energy bounds for the Skyrme and Faddeev models with massive pions, Phys. Lett. B 728, 518 (2014).

[18] M. Gillard, D. Harland, and M. Speight, Skyrmions with low binding energies, Nucl. Phys. B895, 272 (2015).

[19] S. B. Gudnason, Loosening up the Skyrme model, Phys. Rev. D 93, 065048 (2016).

[20] M. F. Atiyah and N. S. Manton, Geometry and kinematics of two skyrmions, Commun. Math. Phys. 153, 391 (1993).

[21] T. Sakai and S. Sugimoto, Low energy hadron physics in holographic QCD, Prog. Theor. Phys. 113, 843 (2005).

[22] L. Bartolini, S. Bolognesi, and A. Proto, From the SakaiSugimoto model to the generalized Skyrme model, Phys. Rev. D 97, 014024 (2018).

[23] C. Adam and A. Wereszczynski, Topological energy bounds in generalized Skyrme models, Phys. Rev. D 89, 065010 (2014).

[24] M. Gillard, D. Harland, E. Kirk, B. Maybee, and M. Speight, A point particle model of lightly bound skyrmions, Nucl. Phys. B917, 286 (2017).

[25] S. Baldino, S. Bolognesi, S. B. Gudnason, and D. Koksal, Solitonic approach to holographic nuclear physics, Phys. Rev. D 96, 034008 (2017).
[26] S. B. Gudnason and M. Nitta, Modifying the pion mass in the loosely bound Skyrme model, Phys. Rev. D 94, 065018 (2016).

[27] S. B. Gudnason, B. Zhang, and N. Ma, Generalized Skyrme model with the loosely bound potential, Phys. Rev. D 94, 125004 (2016).

[28] C. Barnes and N. Turok, A technique for calculating quantum corrections to solitons, arXiv:hep-th/9711071.

[29] C. J. Halcrow, Vibrational quantisation of the $B=7$ Skyrmion, Nucl. Phys. B904, 106 (2016).

[30] S. B. Gudnason and C. Halcrow, $B=5$ Skyrmion as a twocluster system, Phys. Rev. D 97, 125004 (2018).

[31] R. F. Dashen, B. Hasslacher, and A. Neveu, Nonperturbative methods and extended hadron models in field theory 2 . Two-dimensional models and extended hadrons, Phys. Rev. D 10, 4130 (1974).

[32] As long as the Skyrmion is not emanating from a black hole horizon [33,34], we could also take $c_{6}>0$ (for scaling stability due to Derrick's theorem [35]) and $c_{4} \geq 0$; in this paper, however, we will fix $c_{4}>0$.

[33] S. B. Gudnason, M. Nitta, and N. Sawado, Black hole Skyrmion in a generalized Skyrme model, J. High Energy Phys. 09 (2016) 055.

[34] C. Adam, O. Kichakova, Y. Shnir, and A. Wereszczynski, Hairy black holes in the general Skyrme model, Phys. Rev. D 94, 024060 (2016).

[35] G. H. Derrick, Comments on nonlinear wave equations as models for elementary particles, J. Math. Phys. (N.Y.) 5, 1252 (1964).

[36] A. Vilenkin and E. P. S. Shellard, Cosmic Strings and Other Topological Defects, Cambridge Monographs (Cambridge University Press, Cambridge, England, 2000).

[37] G. S. Adkins, C. R. Nappi, and E. Witten, Static properties of nucleons in the Skyrme model, Nucl. Phys. B228, 552 (1983).

[38] Although in the form displayed here the contribution due to the (sextic) BPS-Skyrme term appears with a relative minus sign, all terms contribute to the tensors with the same sign (positive for $U$ and $V$, while $W$ vanishes for the 4-Skyrmion).

[39] In this paper, we use the term "cubic symmetry" to refer loosely to the symmetry of the cube, which is octahedral symmetry. This is because the dual of a cube is a octahedron and the two latter objects share the symmetry: octahedral symmetry. Throughout the paper, we use both terms interchangeably.

[40] K. A. Olive et al. (Particle Data Group), Review of particle Physics, Chin. Phys. C 38, 090001 (2014).

[41] B. R. Barrett, P. Navratil, and J. P. Vary, Ab initio no core shell model, Prog. Part. Nucl. Phys. 69, 131 (2013).

[42] C. Adam, J. Sanchez-Guillen, and A. Wereszczynski, On the spin excitation energy of the nucleon in the Skyrme model, Int. J. Mod. Phys. E 25, 1650097 (2016).

[43] In nuclear matter, the rigid-body quantization can be neglected, and hence the binding energy of nuclear matter gives an upper bound on the mass contribution of the spin to the nucleon, $\tilde{\epsilon}_{1} \lesssim 16 \mathrm{MeV}$.

[44] J. M. Speight, A simple mass-splitting mechanism in the Skyrme model, Phys. Lett. B 781, 455 (2018). 